\title{
PRELIMINARY STUDY ON EPIGEAL INVERTEBRATES FAUNA IN EXPERIMENTAL PEPPER CROPS AT SCDL BUZĂU, ROMANIA
}

\author{
Ioana Florescu ${ }^{1}$, Andrei Teodoru ${ }^{1}$, Alina Gabriela Geicu ${ }^{1}$, Andrei Chiriloaie-Palade ${ }^{1}$, Viorel \\ Fătu ${ }^{1}$, Traian Manole ${ }^{1}$, Toma Mitel ${ }^{2}$ Emilian Mirea ${ }^{2}$,Vasilica Manea ${ }^{2}$, Alexandru Toader ${ }^{2}$, \\ Bogdan Staicu ${ }^{2}$, Floarea Burnichi ${ }^{2}$, Constantina Chireceanu ${ }^{{ }^{*}}$
}

${ }^{1}$ Research-Development Institute for Plant Protection Bucharest, Romania

${ }^{2}$ Research and Development Station for Vegetables Growing Buzau, Romania

${ }^{*}$ Correspondence address:

Research-Development Institute for Plant Protection

8 Ion Ionescu de la Brad 013813, Bucharest, Romania

phone: 004-021-2693231

fax: 004-021-2693239

Email: cchireceanu@yahoo.com

http://www.doi.org/10.54574/RJPP.14.07

\begin{abstract}
This paper presents the species composition and abundance of epigeal invertebrate fauna collected from experimental pepper crops at the Vegetable Research and Development Station Buzău, Romania during 219-2020. The open field bell pepper Buzau 10 (2019) and Cantemir (2020) varieties were used in the experiment in five variants with two factors, diatomite (three doses) and bioinoculant Trichoderma asperellum Td85 strain. Diatomite was local from the Pătârlagele deposit (Buzău County) and antagonistic fungus was from the collection of microorganisms of Research and Development Institute for Plant Protection Bucharest. The fauna on the soil surface was collected using Barber traps, replaced every two weeks from June to October. The ecological parameters of abundance, dominance, constancy and ecological significance have been calculated using specific formulas. Specific attributes of the two products, diatomite and bioinoculant Trichoderma, reflected on the ecological and structural characteristics of invertebrate communities.
\end{abstract}

Key words: pepper crops, invertebrates fauna, ecological parameters, diatomite, bioinoculant Trichoderma

\section{INTRODUCTION}

Epigeal organisms are considered to be micro- and macroinvertebrates that live on the surface and upper layers of the soil, on litter layer and $O$ horizon (Coyle et. al, 2017). Invertebrates contribute to soil formation through the conversion of organic matter on the soil surface into soil, thus supporting functions of the ecosystems (Karuppaiah et al., 2012; Sangle et al., 2015). Soil invertebrate and microorganism communities are influenced by vegetation through the abundance, quality and distribution of organic resources that it produces in both space and time (Giller et al., 1996). According to Bardgett \& Cook (1998), fauna is usually larger on the surface of the soil than in lower horizons. Larger invertebrates are adapted to an open and dry habitat and generally predominate the surface soil and litter layer (Bardgett \& Cook, 1998).

In the last years, studies on epigeal fauna in agriculture crops represented an important concern for many specialists in Romania. Data on various parameters that characterize epigeal fauna in different agricultural crops were published by Manole et al. (2009), Bucur \& Roșca (2011), Buşmachiu \& Bacal (2012) and Rotari (2012) for pepper, eggplant and tomato crops, Mocanu et al. (2017) for wheat crops, Varvara $(2007,2016)$ for sugar beet, sunflower, maize, potato crops, apple orchards and vineyards and Moise (2019) for meadow.

The aim of this study was to assess the epigeal invertebrates, harmful and useful, in experimental pepper crops under influence of treatments with diatomite and bioinoculant Trichoderma asperellum Td85. 


\section{MATERIALS AND METHODS}

The study was carried out in the experimental field of the Vegetable Research and Development Station Buzău (VRDS Buzău), Romania (45.160611 N/26.826609 E/91m) in 2019 and 2020. The open field bell pepper (Capsicum annuum L.), Buzau 10 (2019) and Cantemir (2020) varieties were used in the experiment, in five variants with four repetitions each ( $7 \mathrm{mp}$ per repetition), completed randomized block method: untreated V1, three doses of diatomite V2 (52.5 g), V3 (105 g), V4 (210 g) and V5 (bioinoculant Trichoderma asperellum Td85 strain, 3 grams at the root of the seedling at planting in early June). The diatomite was from the Pătârlagele deposit (Buzău County) and the antagonistic fungus Trichoderma from the collection of microorganisms belonging to the Research and Development Institute for Plant Protection Bucharest. The diatomite was mechanically processed to obtain particles that reach sub-micron dimensions creating amorphous powders with specific properties of nanomaterials. This was used in vegetation season, as powder in the three doses set out above, on rows of plants in June, followed by treating the plants and soil with a dispersion containing diatomite particles in July. The dispersion was achieved by dissolving $83 \mathrm{~g}$ of carboxymethylcellulose in $10 \mathrm{~L}$ of water, followed by the addition of $10 \mathrm{~g}$ of diatomite. After homogenization, the dispersion was transformed into a gel of liquid consistency, but able to keep the diatomite particles in suspension.

To capture the epigeal arthropods fauna on the soil surface, one Barber trap per repetition was used from June to October each year. A 4\% dish soap solution was used into the traps. The biological material collected at 2 weeks interval and conserved in $70 \%$ ethanol was analysed in the Entomology Laboratory of the Research and Development Institute for Plant Protection Bucharest.

The ecological parameters consisting of abundance $(A)$, dominance $(\mathrm{D} \%)$, constancy $(\mathrm{C} \%)$ and ecological significance (W\%) were calculated according to Baban (2006), as follows:

$\mathrm{D} \%=\frac{A \times 100}{n}$, where: A - number of individuals for a species; $\mathrm{n}$ - total number of individuals for all species. According to the values of this parameter, species were classified as subreceding species $(\mathrm{D} 1<1 \%)$, receding species $(\mathrm{D} 2=1-2 \%)$, subdominant species $(\mathrm{D} 3=2-$ $5 \%$ ), dominant species (D4 $=5-10 \%$ ) and eudominant species (D5 $>10 \%$ ).

$\mathrm{C} \%=\frac{n s \times 100}{n}$, where: $\mathrm{ns}$ - number of samples with one species; $\mathrm{n}$ - total number of samples. Depending on the values achieved, species were grouped as accidental $(\mathrm{C} 1=1-25 \%)$, accessory $(\mathrm{C} 2=25-50 \%)$, constant $(\mathrm{C} 3=50-75 \%)$ and euconstant $(\mathrm{C} 4=75-100 \%)$.

$\mathrm{W} \%=\frac{D \times C}{100}$, According to ecological significance, species were ranked as accidental (W1 $<1 \%$ ), accessory $(\mathrm{W} 2=1-5 \%)$ and characteristic $(\mathrm{W} 3>5 \%)$.

\section{RESULTS AND DISCUSSIONS}

A total of 3089 specimens were collected, of which 1455 specimens in 2019 belonging to 3 phyla and 7 classes of invertebrates and 1634 specimens in 2020 belonging to 3 phyla and 8 classes of invertebrates: Phylum: Annelida (Class: Clitellata), Mollusca (Class: Gastropoda), Arthropoda (Class: Malacostraca, Chilopoda, Arachnida, Diplopoda, Entognatha, Insecta) (Table 1). The highest values of numerical abundance had Insecta (63.5\% in 2019 and 65.39\% in 2020) followed by Arachnida (20.72\% in 2019 and $27.82 \%$ in 2020). Among the insects, the order Coleoptera (24.3\%) followed by Diptera $(23.95 \%)$ and Hemiptera $(7.92 \%)$ reached the highest value in 2019, while in 2020, the orders with the 
highest number of individuals were Diptera (20.25\%), Coleoptera (19.03\%) and Hymenoptera $(15.85 \%)$.

Table 1. Taxonomic structure of invertebrates collected in pepper experiment in 2019 and 2020

\begin{tabular}{|c|c|c|c|c|c|c|}
\hline & Phylum & Class & Order & Family & Genus & Species \\
\hline 2019 & 3 & 7 & 17 & 55 & 93 & 117 \\
\hline 2020 & 3 & 8 & 16 & 58 & 104 & 124 \\
\hline
\end{tabular}

From species analysis, the highest five abundances in 2019 were of Agromyza nana (110 specimens), Harpalus pubescens (76 specimens), Poecilus cupreus (72 specimens), Entomobryia arborea (70 specimens) and Chlorops pumilionis (67 specimens). In 2020, the most abundant species were Formica rufa (124 specimens), Agromyza nana (105 specimens), Ceratophysella bengtssoni (87 specimens), Psammotettix striatus (79 specimens), Pseudoophonus rufipes (76 specimens).

Results on total epigeal invertebrate fauna, pest and useful, captured in 2019 and 2020 are presented in Figures 1 and 2, and also in Tables 2 and 3. Data from each year of collection showed differences between variants, both in term of the number of specimens and in terms of the number of taxa (species, genus, family, subfamily) that form the epigean fauna.

The distribution of the fauna in 2019, on functional components harmful and useful, is presented in Figure 1 and Table 2.

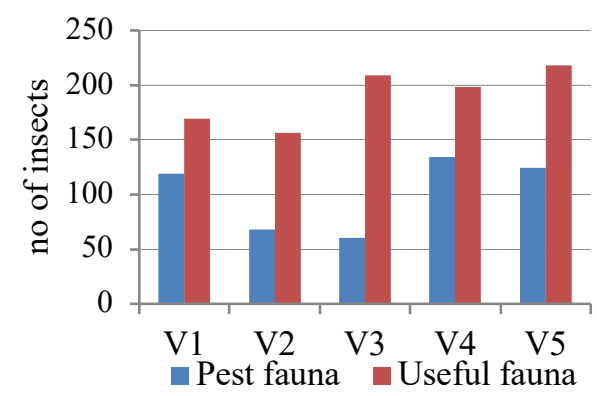

Table 2. Total fauna and number of taxa in pepper variants in 2019

\begin{tabular}{|c|c|c|c|c|c|c|}
\hline \multicolumn{2}{|l|}{ Variant } & V1 & V2 & V3 & V4 & V5 \\
\hline \multicolumn{2}{|l|}{ Total fauna } & 288 & 224 & 269 & 332 & 342 \\
\hline \multicolumn{2}{|l|}{ Pest Fauna (\%) } & 41.4 & 30.35 & 22.30 & 40.36 & 36.25 \\
\hline \multicolumn{2}{|l|}{ Useful Fauna (\%) } & 58.6 & 69.64 & 77.69 & 59.63 & 63.74 \\
\hline \multirow{2}{*}{$\begin{array}{l}\text { No of taxa } \\
\text { (species, genera) }\end{array}$} & PF & 30 & 19 & 13 & 28 & 23 \\
\hline & UF & 47 & 40 & 35 & 38 & 39 \\
\hline
\end{tabular}

Figure 1. Graphic representation of epigean fauna in pepper field in 2019

Compared to the control variant V1, the total number of fauna (harmful and useful) was lower in variants V2 and V3 in which diatomite was applied at a doses of $52.5 \mathrm{~g}$ and $105 \mathrm{~g}$ respectively, and higher in V4 in which $210 \mathrm{~g}$ diatomite was applied and in V5 in which $T$. asperellum Td85 was used at planting (Figure 1).

At the level of variants with increasing doses of diatomite, the pest fauna (PF), compared to control V1, was 1.75 times lower in V2 and 1.98 times lower in V3, and 1.12 times higher in V4. The known insecticidal action of diatomite was observed only in two of the three variants.

In variant V5 in which $T$. asperellum $\mathrm{Td} 85$ was used, both categories of fauna, harmful and useful, were numerically superior to the control V1. This situation can be attributed to the fact that the fungus after application created conditions with favourable effects for the activity of the fauna at ground level.

In all variants of the experiment, the useful fauna (UF) was superior to the pest one, the biggest differences being in V3, V2 and V5 (Table 2). Both PF and UF were represented by a different number of taxa in variants. The number of UF taxa was higher than PF in all variants, the biggest differences being in V3 and V5. 
The distribution of the fauna in 2020, on functional components harmful and useful, is presented in Figure 2 and Table 3.

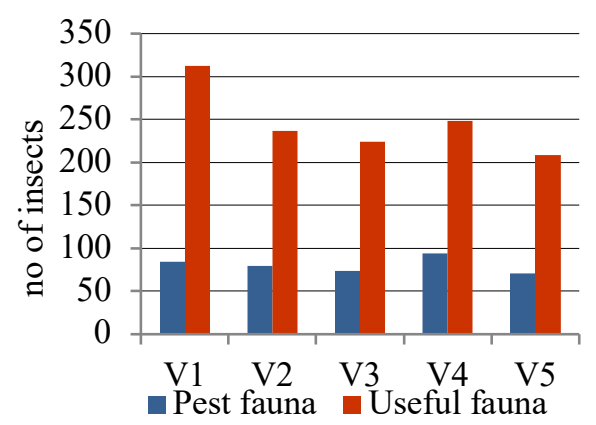

Table 3. Total fauna and number of taxa in pepper variants in 2020

\begin{tabular}{|l|c|c|c|c|c|c|}
\hline Variant & V1 & V2 & V3 & V4 & V5 \\
\hline \multicolumn{2}{|l|}{ Total fauna, dc: } & 396 & 318 & 298 & 342 & 280 \\
\hline \multicolumn{2}{|l|}{ Pest Fauna (\%) } & 21.21 & 25.47 & 24.83 & 27.49 & 25.36 \\
\hline \multicolumn{2}{|l|}{ Useful Fauna (\%) } & 78.79 & 74.53 & 75.17 & 72.51 & 74.64 \\
\hline $\begin{array}{l}\text { No of taxa } \\
\text { (species, genera) }\end{array}$ & P F & 16 & 21 & 21 & 18 & 16 \\
\cline { 2 - 7 } & UF & 34 & 36 & 44 & 50 & 41 \\
\hline
\end{tabular}

Figure 2. Graphic representation of epigean fauna in pepper field in 2020

In contrast to the untreated variant V1, the total fauna (pest and useful) was lower in all other variants. Among the variants with treatments, the total fauna was lower in V5 in which Td85 was used, 3 grams/plant at root during planting, in V3 and V2 in which $105 \mathrm{~g}$ and $52.5 \mathrm{~g}$ of diatomite was applied respectively (Figure 2).

The useful fauna had a situation similar with the total fauna, this being 1.32 times lower in V2, 1.39 times lower in V3, 1.26 times lower in V4 and 1.5 times lower in V5 compared to the control variant. The reduction effect in variants with diatomite could be attributed to the known insecticidal action of the administered diatomite. The reduction in fauna in V5 was unpredictable.

The pest fauna (phytophagous) had lower values in variants V5 (Td85), V3 (diatomite $105 \mathrm{~g} /$ variant) and V2 (diatomite $52.5 \mathrm{~g} /$ variant) compared to the untreated control variant. In V4 (diatomite $210 \mathrm{~g} /$ variant), the pest fauna exceeded that of the control variant 1.2 times (Figure 2).

At the level of variants with diatomite, most specimens (total, pest, useful) were in variant with the highest dose of diatomite. In all variants, the useful fauna was superior to the pest fauna, the UF/PF ratio being approximately 3:1 (Table 3). Both PF and UF were represented by a different number of taxa in variants. The number of UF taxa was higher than the PF taxa in all variants (Table 3) confirming a greater richness of zoophagous species than phytophagous pest species.

The ecological parameters represented by abundance $(A)$, dominance $(\mathrm{D} \%)$, constancy $(\mathrm{C} \%)$ and ecological significance $(\mathrm{W} \%)$ calculated for pest and useful faunistic components for the two years study are presented in Tables 4, 5, 6 and 7. 
Table 4. List of the identified taxa and ecological parameters for pest fauna in 2019

\begin{tabular}{|c|c|c|c|c|c|c|c|c|c|c|c|c|c|c|c|c|c|c|c|c|}
\hline \multirow{2}{*}{$\begin{array}{c}\text { Taxa of pest fauna } \\
2019\end{array}$} & \multicolumn{4}{|c|}{ V1 } & \multicolumn{4}{|c|}{ V2 } & \multicolumn{4}{|c|}{$\mathrm{V3}$} & \multicolumn{4}{|c|}{ V4 } & \multicolumn{4}{|c|}{ V5 } \\
\hline & $\mathbf{A}$ & D(\%) & $\mathrm{C}(\%)$ & $w(\%)$ & A & D(\%) & $\mathrm{C}(\%)$ & $\mathrm{W}(\%)$ & $\mathbf{A}$ & $\mathrm{D}(\%)$ & $\mathbf{C}(\%)$ & $w(\%)$ & $\mathbf{A}$ & D(\%) & $\mathrm{C}(\%)$ & $\begin{array}{c}\mathbf{W} \\
(\%)\end{array}$ & $\mathbf{A}$ & $\mathbf{D}(\%)$ & $\mathrm{C}(\%)$ & W(\%) \\
\hline Acari & 4 & 3,37 & 33,34 & 1,12 & 0 & 0 & 0 & 0 & 0 & 0 & 0 & 0 & 3 & 2,24 & 16,67 & 0,37 & 2 & 1,62 & 16,67 & 0,27 \\
\hline Acyrtosiphon pisum Har. & 0 & 0 & 0 & 0 & 0 & 0 & 0 & 0 & 0 & 0 & 0 & 0 & 2 & 1,50 & 16,67 & 0,25 & 1 & 0,81 & 16,67 & 0,13 \\
\hline Gastropoda & 1 & 0,84 & 16,67 & 0,14 & 0 & 0 & 0 & 0 & 0 & 0 & 0 & 0 & 1 & 0,75 & 16,67 & 0,12 & 0 & 0 & 0 & $\left.\frac{0}{0}+30\right)$ \\
\hline Aphis fabae Scop. & 3 & 2,53 & 33,34 & 0,84 & 3 & 4,42 & 33,34 & 1,47 & 2 & 3,34 & 16,67 & 0,55 & 1 & 0,75 & 16,67 & 0,12 & 2 & 1,62 & 33,34 & 0,54 \\
\hline Agromyza flaviceps Fall. & 8 & 6,73 & 83,34 & 5,60 & 6 & 8,83 & 33,34 & 2,94 & 1 & 1,67 & 16,67 & 0,27 & 8 & 5,97 & 33,34 & 1,99 & 12 & 9,68 & 50,00 & 4,84 \\
\hline Agromyza nana Meig. & 30 & 25,21 & 50,00 & 12,60 & 15 & 22,06 & 100 & 22,06 & 17 & 28,34 & 50,00 & 14,17 & 30 & 22,39 & 83,34 & 18,65 & 18 & 14,52 & 66,67 & 9,68 \\
\hline Aphtona pygmaea Kutsch. & 0 & 0 & 0 & 0 & 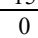 & 0 & 0 & 0 & 0 & 0 & 0 & 0 & $\frac{2}{2}$ & 1,50 & 16,67 & 0,25 & 0 & 0 & 0 & 0 \\
\hline Attagenus pellio $L$. & 0 & 0 & 0 & 0 & 0 & 0 & 0 & 0 & 0 & 0 & 0 & 0 & 1 & 0,75 & 16,67 & 0,12 & 1 & 0,81 & 16,67 & 0,13 \\
\hline Austroagallia sinuata Muls. et Rey & 1 & 0,84 & 16,67 & 0,14 & 2 & 2,95 & 16,67 & 0,49 & 1 & 1,67 & 16,67 & 0,27 & 3 & 2,24 & 33,34 & 0,74 & 3 & 2,42 & 16,67 & 0,40 \\
\hline Chaetocnema tibialis Illig. & 2 & 1,68 & 16,67 & 0,28 & 0 & 0 & 0 & 0 & 0 & 0 & 0 & 0 & 1 & 0,75 & 16,67 & 0,12 & 0 & 0 & 0 & 0 \\
\hline Chlorops pumilionis Bjerk. & 10 & 8,41 & 16,67 & 1,40 & 2 & 2,95 & 16,67 & 0,49 & 0 & 0 & 0 & 0 & 22 & 16,42 & 16,67 & 2,73 & 33 & 26,62 & 50,00 & 13,31 \\
\hline Cicadella viridis $L$. & 3 & 2,53 & 16,67 & 0,42 & 2 & 2,95 & 33,34 & 0,98 & 0 & 0 & 0 & 0 & 0 & $\frac{107}{0}$ & 0 & 0 & $\frac{2}{2}$ & 1,62 & 16,67 & 0,27 \\
\hline Delia antiqua Meig. & 1 & 0,84 & 16,67 & 0,14 & 0 & 0 & 0 & 0 & 0 & 0 & 0 & 0 & 0 & 0 & 0 & 0 & 0 & 0 & 0 & 0 \\
\hline Diptera-Chloropidae & 3 & 2,53 & 33,34 & 0,84 & 4 & 5,89 & 33,34 & 1,96 & 0 & 0 & 0 & 0 & 4 & 2,99 & 16,67 & 0,49 & 1 & 0,81 & 16,67 & 0,13 \\
\hline Diptera-Muscidae & 26 & 21,85 & 66,67 & 14,56 & 21 & 30,89 & 50,00 & 15,44 & 27 & 45,00 & 66,67 & 30,00 & 10 & 7,47 & 83,34 & 6,22 & 14 & 11,29 & 50,00 & 5,64 \\
\hline Diptera - Sciaridae & $\frac{20}{5}$ & 4,21 & 50,00 & 2,10 & $\frac{11}{3}$ & 4,42 & 33,34 & 1,47 & 2 & 3,34 & 33,34 & 1,11 & 11 & 8,21 & 66,67 & $\begin{array}{l}5,22 \\
5,47\end{array}$ & 9 & 7,26 & 50,00 & 3,63 \\
\hline Diptera - Simuliidae- Simulium sp. & 1 & 0,84 & 16,67 & 0,14 & 0 & 0 & 0 & 0 & 2 & 3,34 & 16,67 & 0,55 & 3 & 2,24 & 16,67 & 0,37 & 0 & 0 & 0 & 0 \\
\hline Diptera - Trypetidae & 1 & 0,84 & 16,67 & 0,14 & 0 & 0 & 0 & 0 & 0 & 0 & 0 & 0 & 0 & 0 & 0 & 0 & 0 & 0 & 0 & 0 \\
\hline Dolycoris baccarum $L$. & 1 & 0,84 & 16,67 & 0,14 & 0 & 0 & 0 & 0 & 0 & 0 & 0 & 0 & 0 & 0 & 0 & 0 & 0 & 0 & 0 & 0 \\
\hline Elachiptera cornuta Fall. & $\frac{1}{2}$ & 1,68 & 33,34 & 0,56 & 1 & 1,47 & 16,67 & 0,24 & 0 & 0 & 0 & 0 & 2 & 1,50 & 16,67 & 0,25 & 6 & 4,84 & 33,34 & 1,61 \\
\hline Empoasca solani Curt. & 0 & 0 & 0 & 0 & 0 & 0 & 0 & 0 & 0 & 0 & 0 & 0 & 6 & 4,48 & 33,34 & 1,49 & 0 & 0 & 0 & 0 \\
\hline Halticus apterus $L$. & 1 & 0,84 & 16,67 & 0,14 & 0 & 0 & 0 & 0 & 0 & 0 & 0 & 0 & 0 & 0 & 0 & 0 & 0 & 0 & 0 & 0 \\
\hline Haltica oleracea $L$. & 2 & 1,68 & 16,67 & 0,28 & 0 & 0 & 0 & 0 & 0 & 0 & 0 & 0 & 0 & 0 & 0 & 0 & 0 & 0 & 0 & 0 \\
\hline Helicella candicans Pfeiff. & 2 & 1,68 & 16,67 & 0,28 & 0 & 0 & 0 & 0 & 0 & 0 & 0 & 0 & 0 & 0 & 0 & 0 & 0 & 0 & 0 & 0 \\
\hline Hemitarsonemus latus Banks. & 1 & 0,84 & 16,67 & 0,14 & 1 & 1,47 & 16,67 & 0,24 & 0 & 0 & 0 & 0 & 4 & 2,99 & 33,34 & 0,99 & 0 & 0 & 0 & 0 \\
\hline Hippelates plebejus Loew. & 0 & $\frac{0}{0}$ & 0 & $\frac{0}{0}$ & 0 & 0 & 0 & $\frac{0}{0}$ & 0 & 0 & 0 & 0 & 1 & 0,75 & 16,67 & 0,12 & 0 & 0 & 0 & $\frac{0}{0}$ \\
\hline Kakothrips robustus Uzel & 1 & 0,84 & 16,67 & 0,14 & 1 & 1,47 & 16,67 & 0,24 & 0 & 0 & 0 & 0 & 0 & 0 & 0 & 0 & 0 & 0 & 0 & 0 \\
\hline Lepidoptera & 0 & 0 & $\frac{0}{0}$ & 0 & 0 & 0 & 0 & $\frac{0}{0}$ & 1 & 1,67 & 16,67 & 0,27 & 0 & 0 & 0 & 0 & 0 & 0 & 0 & 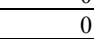 \\
\hline Limotettix striola Fall. & $\frac{0}{2}$ & 1,68 & 33,34 & 0,56 & 0 & 0 & 0 & 0 & $\frac{1}{2}$ & 3,34 & 16,67 & 0,27 & $\frac{0}{1}$ & 0,75 & 16,67 & 0,12 & $\frac{0}{5}$ & 4,04 & 16,67 & 0,67 \\
\hline Lygus pratensis & 0 & 0 & 0 & $\frac{0}{0}$ & 1 & 1,47 & 16,67 & 0,24 & 0 & 0 & 0 & $\frac{0}{0}$ & $\frac{1}{1}$ & 0,75 & 16,67 & 0,12 & 1 & 0,81 & 16,67 & 0,13 \\
\hline Longitarsus luridus Scop. & 0 & 0 & 0 & 0 & 1 & 1,47 & 16,67 & 0,24 & 0 & 0 & 0 & 0 & 0 & 0 & 0 & 0 & 0 & 0 & 0 & 0 \\
\hline $\begin{array}{l}\text { Longitarsus pratensis Panz. } \\
\text {. }\end{array}$ & 1 & 0,84 & 16,67 & 0,14 & $\frac{1}{0}$ & $\frac{1,+1}{0}$ & 0 & $\frac{0,24}{0}$ & 0 & 0 & 0 & 0 & 0 & 0 & 0 & 0 & 0 & 0 & 0 & 0 \\
\hline Lucilia sericata Meig. & 0 & 0 & 0 & 0 & 0 & 0 & 0 & 0 & 1 & 1,67 & 16,67 & 0,27 & 2 & 1,50 & 16,67 & 0,25 & 1 & 0,81 & 16,67 & 0,13 \\
\hline Macrosiphum gei Koch. & 0 & 0 & 0 & 0 & 1 & 1,47 & 16,67 & 0,24 & 0 & 0 & 0 & 0 & 4 & 2,99 & 16,67 & 0,49 & 2 & 1,62 & 16,67 & 0,27 \\
\hline Meromyza nigriventris Macq. & 1 & 0,84 & 16,67 & 0,14 & 0 & 0 & 0 & 0 & 0 & 0 & 0 & 0 & 2 & 1,50 & 16,67 & 0,25 & 2 & 1,62 & 16,67 & 0,27 \\
\hline Mythimna l-album $L$. & 0 & 0 & 0 & 0 & 0 & 0 & 0 & 0 & 0 & 0 & 0 & 0 & 1 & 0,75 & 16,67 & 0,25 & 0 & 0 & 0 & 0 \\
\hline Orthocephalus coriaceus $F$. & 0 & 0 & 0 & 0 & 0 & 0 & 0 & 0 & $\frac{0}{1}$ & 1,67 & 16,67 & 0,27 & $\frac{1}{1}$ & 0,75 & 16,67 & 0,25 & $\frac{0}{1}$ & 0,81 & 16,67 & 0,13 \\
\hline Phyllotreta atra $F$. & 0 & 0 & 0 & 0 & 0 & 0 & 0 & 0 & $\frac{1}{0}$ & $\frac{107}{0}$ & 0 & $\frac{0,21}{0}$ & $\frac{1}{0}$ & $\frac{0}{0}$ & $\frac{10,0}{0}$ & $\frac{0}{0}$ & $\frac{1}{2}$ & 1,62 & 16,67 & 0,17 \\
\hline Phyllotreta vittula Redtb. & 1 & 0,84 & 16,67 & 0,14 & 0 & 0 & 0 & 0 & 0 & 0 & 0 & 0 & 2 & 1,50 & 33,34 & 0,50 & 0 & 0 & 0 & 0 \\
\hline Phytomyza lonicerae Rob.-Desv. & 1 & 0,84 & 16,67 & 0,14 & 1 & 1,47 & 16,67 & 0,24 & 0 & 0 & 0 & 0 & 0 & 0 & 0 & 0 & 0 & 0 & 0 & 0 \\
\hline Psammotettix striatus $L$. & 1 & 0,84 & 16,67 & 0,14 & 0 & $\frac{0}{0}$ & 0 & 0 & 0 & 0 & 0 & 0 & 0 & 0 & 0 & 0 & 0 & 0 & 0 & 0 \\
\hline Psylloides chrysocephala $L$. & 1 & 0,07 & 16,67 & 0,14 & 1 & 1,47 & 16,67 & 0,24 & $\frac{3}{2}$ & 3,34 & 33,34 & 1,11 & $\frac{0}{4}$ & 2,99 & 50,00 & $\frac{0}{1,49}$ & 0 & 0 & 0 & $\frac{0}{0}$ \\
\hline Rhinoncus pericarpius $L$. & $\frac{1}{2}$ & 1,68 & 16,67 & 0,28 & 0 & 0 & 0 & $\frac{0}{0}$ & 0 & 0 & 0 & $\frac{114}{0}$ & 0 & 0 & 0 & $\frac{17}{0}$ & 0 & 0 & 0 & 0 \\
\hline Scatophaga stercoraria L. & 0 & 0 & $\frac{0}{0}$ & 0 & 0 & 0 & 0 & 0 & 1 & 1,67 & 16,67 & 0,27 & 1 & 0,75 & 16,67 & 0,25 & 0 & 0 & 0 & 0 \\
\hline Tingis reticulata Herr-Scaff. & 0 & 0 & 0 & 0 & 1 & $\begin{array}{c}1,47 \\
\end{array}$ & 16,67 & 0,24 & 0 & $\frac{1}{0}$ & $\frac{10}{0}$ & $\frac{0}{0}$ & 0 & 0 & $\frac{10}{0}$ & $\frac{0}{0}$ & 0 & 0 & 0 & 0 \\
\hline
\end{tabular}




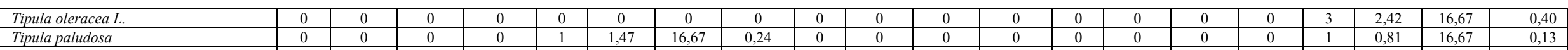
\begin{tabular}{|l|c|c|c|c|c|c|c|c|c|c|c|c|c|c|c|c|c|c|c|c|c|c|c|c|c|c|}
\hline Tipula paludosa & 0 & 0 & 0 & 0 & 1 & 1,47 & 16,67 & 0,24 & 0 & 0 & 0 & 0 & 0 & 0 & 0 & 0 & 1 & 0,81 & 16,67 & 0,13 \\
\hline Xanthocrambus saxonellus Zinck. & 0 & 0 & 0 & 0 & 0 & 0 & 0 & 0 & 0 & 0 & 0 & 0 & 0 & 0 & 0 & 0 & 2 & 1,62 & 16,67 & 0,27 \\
\hline
\end{tabular}

Table 5. List of the identified taxa and ecological parameters for useful fauna in 2019

\begin{tabular}{|c|c|c|c|c|c|c|c|c|c|c|c|c|c|c|c|c|c|c|c|c|}
\hline \multirow{2}{*}{$\begin{array}{c}\text { Taxa of useful fauna } \\
2019\end{array}$} & \multicolumn{4}{|c|}{ V1 } & \multicolumn{4}{|c|}{ V2 } & \multicolumn{4}{|c|}{ V3 } & \multicolumn{4}{|c|}{ V4 } & \multicolumn{4}{|c|}{ V5 } \\
\hline & $\mathbf{A}$ & D(\%) & $\mathrm{C}(\%)$ & $\mathrm{W}(\%)$ & $\mathbf{A}$ & $\mathrm{D}(\%)$ & $\mathrm{C}(\%)$ & $\mathrm{W}(\%)$ & $\mathbf{A}$ & D(\%) & $\mathrm{C}(\%)$ & $\mathrm{W}(\%)$ & $\mathbf{A}$ & $\mathrm{D}(\%)$ & $\mathrm{C}(\%)$ & W(\%) & $\mathbf{A}$ & $\mathrm{D}(\%)$ & $\mathrm{C}(\%)$ & $\mathrm{W}(\%)$ \\
\hline Acupalpus elegans Dej. & 1 & 0,60 & 16,67 & 0,10 & 0 & 0 & 0 & 0 & 0 & 0 & 0 & 0 & 2 & 1,01 & 33,34 & 0,33 & 0 & 0 & 0 & 0 \\
\hline Aleochara moerens Gyll. & 1 & 0,60 & 16,67 & 0,10 & 0 & 0 & 0 & 0 & 0 & 0 & 0 & 0 & 0 & 0 & 0 & 0 & 1 & 0,46 & 16,67 & 0,07 \\
\hline Aleochara ruficornis Grav. & 0 & 0 & 0 & 0 & 1 & 0,65 & 16,67 & 0,10 & 0 & 0 & 0 & 0 & 0 & 0 & 0 & 0 & 0 & 0 & 0 & 0 \\
\hline Aleochara laevigata Gyll. & 1 & 0,60 & 16,67 & 0,10 & 1 & 0,65 & 16,67 & 0,10 & 0 & 0 & 0 & 0 & 1 & 0,51 & 16,67 & 0,08 & 0 & 0 & 0 & 0 \\
\hline Allolobophora caliginosa Sav. & 0 & 0 & 0 & 0 & 0 & 0 & 0 & 0 & 0 & 0 & 0 & 0 & 2 & 1,01 & 16,67 & 0,16 & 0 & 0 & 0 & 0 \\
\hline Araneae & 40 & 23,67 & 83,34 & 19,72 & 37 & 23,72 & 83,34 & 19,76 & 57 & 27,28 & 83,34 & 22,73 & 66 & 33,34 & 100,0 & 33,34 & 56 & 25,69 & 83,34 & 21,41 \\
\hline Apis melifera & 0 & 0 & 0 & 0 & 0 & 0 & 0 & 0 & 0 & 0 & 0 & 0 & 0 & 0 & 0 & 0 & 1 & 0,46 & 16,67 & 0,07 \\
\hline Anthelephila cyanea Hope & 0 & 0 & 0 & 0 & 1 & 0,65 & 16,67 & 0,10 & 1 & 0,48 & 16,67 & 0,08 & 3 & 1,52 & 33,34 & 0,50 & 0 & 0 & 0 & 0 \\
\hline Anthicus floralis $L$. & 5 & 2,96 & 50,00 & 1,48 & 1 & 0,65 & 16,67 & 0,10 & 0 & 0 & 0 & 0 & 1 & 0,51 & 16,67 & 0,08 & 0 & 0 & 0 & 0 \\
\hline Anthicus (Endomia)melanocephalus Con. & 1 & 0,60 & 16,67 & 0,10 & 1 & 0,65 & 16,67 & 0,10 & 0 & 0 & 0 & 0 & 1 & 0,51 & 16,67 & 0,08 & 1 & 0,46 & 16,67 & 0,07 \\
\hline Amalorrhynchus melanarius Steph. & 2 & 1,19 & 16,67 & 0,19 & 0 & 0 & 0 & 0 & 0 & 0 & 0 & 0 & 0 & 0 & 0 & 0 & 1 & 0,46 & 16,67 & 0,07 \\
\hline Amara crenata Dej. & 0 & 0 & 0 & 0 & 0 & 0 & 0 & 0 & 1 & 0,48 & 16,67 & 0,08 & 0 & 0 & 0 & 0 & 0 & 0 & 0 & 0 \\
\hline Amara apricaria Payk. & 3 & 1,78 & 16,67 & 0,29 & 1 & 0,65 & 16,67 & 0,10 & 0 & 0 & 0 & 0 & 0 & 0 & 0 & 0 & 0 & 0 & 0 & 0 \\
\hline Anthocoris nemorumL. & 8 & 4,74 & 33,34 & 1,58 & 1 & 0,65 & 16,67 & 0,10 & 3 & 1,44 & 33,34 & 0,48 & 12 & 6,06 & 50,00 & 3,03 & 9 & 4,13 & 33,34 & 1,37 \\
\hline Aporrrectodea caliginosa Sav. & 0 & 0 & 0 & 0 & 0 & 0 & 0 & 0 & 2 & 0,96 & 16,67 & 0,16 & 3 & 1,52 & 16,67 & 0,25 & 1 & 0,46 & 16,67 & 0,07 \\
\hline Bembidion assimile Gyll. & 1 & 0,60 & 16,67 & 0,10 & 1 & 0,65 & 16,67 & 0,10 & 0 & 0 & 0 & 0 & 0 & 0 & 0 & 0 & 0 & 0 & 0 & 0 \\
\hline Bembidion properans Steph. & 0 & 0 & 0 & 0 & 0 & 0 & 0 & 0 & 1 & 0,48 & 16,67 & 0,08 & 0 & 0 & 0 & 0 & 0 & 0 & 0 & 0 \\
\hline Blaniulus guttulatus Bosc. & 1 & 0,60 & 16,67 & 0,10 & 4 & 2,57 & 16,67 & 0,42 & 2 & 0,96 & 16,67 & 0,16 & 2 & 1,01 & 16,67 & 0,16 & 4 & 1,84 & 16,67 & 0,30 \\
\hline Cartodere ruficollis Marsh. & 1 & 0,60 & 16,67 & 0,10 & 0 & 0 & 0 & 0 & 0 & 0 & 0 & 0 & 0 & 0 & 0 & 0 & 0 & 0 & 0 & 0 \\
\hline Charopus pallipes Oliv. & 0 & 0 & 0 & 0 & 0 & 0 & 0 & 0 & 0 & 0 & 0 & 0 & 1 & 0,51 & 16,67 & 0,08 & 0 & 0 & 0 & 0 \\
\hline Chilopora rubicunda Er. & 0 & 0 & 0 & 0 & 0 & 0 & 0 & 0 & 0 & 0 & 0 & 0 & 0 & 0 & 0 & 0 & 1 & 0,46 & 16,67 & 0,07 \\
\hline Chromatoiulus unilineatus Koch. & 0 & 0 & 0 & 0 & 0 & 0 & 0 & 0 & 3 & 1,44 & 33,34 & 0,48 & 4 & 2,02 & 16,67 & 0,33 & 3 & 1,38 & 16,67 & 0,23 \\
\hline Chrysoperla carnea $L$. & 0 & 0 & 0 & 0 & 1 & 0,65 & 16,67 & 0,10 & 0 & 0 & 0 & 0 & 0 & 0 & 0 & 0 & 0 & 0 & 0 & 0 \\
\hline Coccinella 5-punctata L. & 1 & 0,60 & 16,67 & 0,10 & 1 & 0,65 & 16,67 & 0,10 & 0 & 0 & 0 & 0 & 0 & 0 & 0 & 0 & 2 & 0,92 & 16,67 & 0,15 \\
\hline Coccinella 7-punctata L. & 0 & 0 & 0 & 0 & 0 & 0 & 0 & 0 & 1 & 0,48 & 16,67 & 0,08 & 0 & 0 & 0 & 0 & 0 & 0 & 0 & 0 \\
\hline Corticaria longicollis Herbst. & 1 & 0,60 & 16,67 & 0,10 & 0 & 0 & 0 & 0 & 0 & 0 & 0 & 0 & 0 & 0 & 0 & 0 & 0 & 0 & 0 & 0 \\
\hline Corticarina fulvipes Com. & 0 & 0 & 0 & 0 & 0 & 0 & 0 & 0 & 1 & 0,48 & 16,67 & 0,08 & 0 & 0 & 0 & 0 & 0 & 0 & 0 & 0 \\
\hline Corticarina gibbosa Herbst. & 1 & 0,60 & 16,67 & 0,10 & 0 & 0 & 0 & 0 & 1 & 0,48 & 16,67 & 0,08 & 1 & 0,51 & 16,67 & 0,08 & 0 & 0 & 0 & 0 \\
\hline Corticarina truncatella Mnnh. & 1 & 0,60 & 16,67 & 0,10 & 0 & 0 & 0 & 0 & 0 & 0 & 0 & 0 & 2 & 1,01 & 16,67 & 0,16 & 0 & 0 & 0 & 0 \\
\hline Diptera-Dolichopodidae & 0 & 0 & 0 & 0 & 0 & 0 & 0 & 0 & 4 & 1,92 & 16,67 & 0,32 & 5 & 2,53 & 16,67 & 0,42 & 3 & 1,38 & 33,34 & 0,46 \\
\hline Dolichovespula saxonica $F$. & 2 & 1,19 & 16,67 & 0,19 & 1 & 0,65 & 16,67 & 0,10 & 0 & 0 & 0 & 0 & 0 & 0 & 0 & 0 & 0 & 0 & 0 & 0 \\
\hline Drasterius bimaculatus Rossi & 1 & 0,60 & 16,67 & 0,10 & 1 & 0,65 & 16,67 & 0,10 & 3 & 1,44 & 33,34 & 0,48 & 0 & 0 & 0 & 0 & 3 & 1,38 & 16,67 & 0,23 \\
\hline Empis livada $L$. & 0 & 0 & 0 & 0 & 0 & 0 & 0 & 0 & 0 & 0 & 0 & 0 & 0 & 0 & 0 & 0 & 2 & 0,92 & 16,67 & 0,15 \\
\hline Entomobryia arborea Tullb. & 1 & 0,60 & 16,67 & 0,10 & 0 & 0 & 0 & 0 & 24 & 11,49 & 16,67 & 1,91 & 23 & 11,62 & 16,67 & 1,93 & 22 & 10,10 & 33,34 & 3,36 \\
\hline Entomobrya multifasciata Tullb. & 0 & 0 & 0 & 0 & 10 & 6,41 & 16,67 & 1,06 & 0 & 0 & 0 & 0 & 0 & 0 & 0 & 0 & 0 & 0 & 0 & 0 \\
\hline Formica rufa $L$. & 2 & 1,19 & 16,67 & 0,19 & 3 & 1,93 & 50,00 & 0,96 & 3 & 1,44 & 50,00 & 0,72 & 1 & 0,51 & 16,67 & 0,08 & 6 & 2,76 & 33,34 & 0,92 \\
\hline Geocoris grylloides $L$. & 0 & 0 & 0 & 0 & 0 & 0 & 0 & 0 & 0 & 0 & 0 & 0 & 0 & 0 & 0 & 0 & 1 & 0,46 & 16,67 & 0,07 \\
\hline Halictus maculatus Smith. & 1 & 0,60 & 16,67 & 0,10 & 0 & 0 & 0 & 0 & 0 & 0 & 0 & 0 & 0 & 0 & 0 & 0 & 0 & 0 & 0 & 0 \\
\hline Harpalus aeneus $F$. & 1 & 0,60 & 16,67 & 0,10 & 1 & 0,65 & 16,67 & 0,10 & 1 & 0,48 & 16,67 & 0,08 & 0 & 0 & 0 & 0 & 0 & 0 & 0 & 0 \\
\hline Harpalus calceatus Duft. & 0 & 0 & 0 & 0 & 0 & 0 & 0 & 0 & 0 & 0 & 0 & 0 & 1 & 0,51 & 16,67 & 0,08 & 2 & $\begin{array}{l}0,92 \\
\end{array}$ & 16,67 & 0,15 \\
\hline Harpalus distinguendus Duft. & 4 & 2,37 & 50,00 & 1,18 & 2 & 1,29 & 33,34 & 0,43 & 11 & 5,27 & 50,00 & 2,63 & 3 & 1,52 & 33,34 & 0,50 & 6 & 2,76 & 33,34 & 0,92 \\
\hline Harpalus griseus Panz. & 4 & 2,37 & 16,67 & 0,39 & 4 & 2,57 & 16,67 & 0,42 & 1 & 0,48 & 16,67 & 0,08 & 0 & 0 & 0 & 0 & 0 & 0 & 0 & 0 \\
\hline
\end{tabular}


Romanian Journal for Plant Protection, Vol. XIV, 2021

ISSN 2248 - 129X; ISSN-L 2248 - 129X

\begin{tabular}{|c|c|c|c|c|c|c|c|c|c|c|c|c|c|c|c|c|c|c|c|c|}
\hline Hemerobius humulinus $L$. & 1 & 0,60 & 16,67 & 0,10 & 1 & 0,65 & 16,67 & 0,10 & 0 & 0 & 0 & 0 & 0 & 0 & 0 & 0 & 0 & 0 & 0 & 0 \\
\hline Hymenoptera - Braconidae & 1 & 0,60 & 16,67 & 0,10 & 0 & 0 & 0 & $\frac{1+0}{0}$ & 1 & 0,48 & 16,67 & 0,08 & 3 & 1,52 & 50,00 & 0,76 & 1 & 0,46 & 16,67 & 0,07 \\
\hline Hymenoptera-Ichneumonidae & 1 & 0,60 & 16,67 & 0,10 & 0 & 0 & 0 & 0 & 0 & 0 & 0 & 0 & 0 & 0 & 0 & 0 & 0 & 0 & 0 & 0 \\
\hline Hymenoptera-Scelionidae & 1 & 0,60 & 16,67 & 0,10 & 1 & 0,65 & 16,67 & 0,10 & 0 & 0 & 0 & 0 & 5 & 2,53 & 33,34 & 0,84 & 4 & 1,84 & 50,00 & 0,92 \\
\hline Ichneumon sp. & 0 & 0 & 0 & 0 & 2 & 1,29 & 16,67 & 0,21 & 0 & 0 & 0 & 0 & 1 & 0,51 & 16,67 & 0,08 & 0 & 0 & 0 & 0 \\
\hline Ischnopoda atra Grav. & 1 & 0,60 & 16,67 & 0,10 & 0 & 0 & 0 & 0 & 0 & 0 & 0 & 0 & 0 & 0 & 0 & 0 & 0 & 0 & 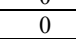 & 0 \\
\hline Lasius flavus $F$. & 0 & $\frac{0}{0}$ & 0 & 0 & 2 & 1,29 & 16,67 & 0,21 & 0 & 0 & 0 & 0 & 0 & 0 & 0 & 0 & 0 & 0 & 0 & 0 \\
\hline Lasius fuliginosus Latr. & 0 & 0 & 0 & 0 & 0 & 0 & 0 & 0 & 0 & 0 & 0 & 0 & 5 & 2,53 & 33,34 & 0,84 & 3 & 1,38 & 33,34 & 0,46 \\
\hline $\begin{array}{l}\text { Lasius niger } L \text {. } \\
\end{array}$ & 0 & 0 & 0 & 0 & 0 & 0 & 0 & 0 & 0 & 0 & 0 & 0 & 1 & 0,51 & 16,67 & 0,08 & 1 & 0,46 & 16,67 & 0,07 \\
\hline Lathrobium elongatum L. & 0 & 0 & 0 & 0 & 2 & 1,29 & 16,67 & 0,21 & 0 & 0 & 0 & 0 & 0 & 0 & 0 & 0 & 0 & 0 & 0 & 0 \\
\hline Lepidocyrtus cyaneus Tullb. & 0 & 0 & 0 & 0 & 0 & 0 & 0 & 0 & 1 & 0,48 & 16,67 & 0,08 & 1 & 0,51 & 16,67 & 0,08 & 2 & 0,92 & 16,67 & 0,15 \\
\hline Lithobius forficatus $L$. & 0 & 0 & 0 & 0 & 1 & 0,65 & 16,67 & 0,10 & 2 & 0,96 & 16,67 & 0,16 & 0 & 0 & 0 & 0 & 0 & 0 & 0 & 0 \\
\hline Myrmica rubra $L$. & 6 & 3,55 & 33,34 & 1,18 & 5 & 3,21 & 66,67 & 2,14 & 3 & 1,44 & 33,34 & 0,48 & 2 & 1,01 & 16,67 & 0,16 & 26 & 11,93 & 66,67 & 7,95 \\
\hline Malachius bipustulatus $L$. & 1 & 0,60 & 16,67 & 0,10 & 0 & 0 & 0 & 0 & 0 & 0 & 0 & 0 & 0 & 0 & 0 & 0 & 0 & 0 & 0 & 0 \\
\hline Nabis ferus L. & 2 & 1,19 & 33,34 & 0,39 & 0 & 0 & 0 & 0 & 0 & 0 & 0 & 0 & 0 & 0 & 0 & 0 & 1 & 0,46 & 16,67 & 0,07 \\
\hline Nabis pseudoferus Remane. & 1 & 0,60 & 16,67 & 0,10 & 2 & 1,29 & 16,67 & 0,21 & 2 & 0,96 & 33,34 & 0,32 & 1 & 0,51 & 16,67 & 0,08 & 1 & 0,46 & 16,67 & 0,07 \\
\hline Ocalea rivularis Mill. & 0 & 0 & 0 & 0 & 0 & 0 & 0 & 0 & 0 & 0 & 0 & 0 & 0 & 0 & 0 & 0 & 1 & 0,46 & 16,67 & 0,07 \\
\hline Ocys quinquestriatus Gyll. & 0 & 0 & 0 & 0 & 0 & 0 & 0 & 0 & 2 & 0,96 & 16,67 & 0,16 & 0 & 0 & 0 & 0 & 0 & 0 & 0 & 0 \\
\hline Orius niger Wolff. & 1 & 0,60 & 16,67 & 0,10 & 0 & 0 & 0 & 0 & 0 & 0 & 0 & 0 & 0 & 0 & 0 & 0 & 0 & 0 & 0 & 0 \\
\hline Oxytelus inustus Grav. & 0 & 0 & 0 & 0 & 0 & 0 & 0 & 0 & 0 & 0 & 0 & 0 & 0 & 0 & 0 & 0 & 1 & 0,46 & 16,67 & 0,07 \\
\hline Phalangium opilio $L$. & 12 & 7,10 & 33,34 & 2,36 & 12 & 7,70 & 33,34 & 2,56 & 4 & 1,92 & 16,67 & 0,32 & 14 & 7,07 & 50,00 & 3,53 & 4 & 1,84 & 33,34 & 0,61 \\
\hline Philonthus aerosus Kiesw. & 2 & 1,19 & 16,67 & 0,19 & 1 & 0,65 & 16,67 & 0,10 & 0 & 0 & 0 & 0 & 0 & 0 & 0 & 0 & 0 & 0 & 0 & 0 \\
\hline Philonthus albipes Grav. & 0 & 0 & 0 & 0 & 0 & 0 & 0 & 0 & 1 & 0,48 & 16,67 & 0,08 & 0 & 0 & 0 & 0 & 0 & 0 & 0 & 0 \\
\hline Phloeonomus monilicornis Gyll. & 0 & 0 & 0 & 0 & 1 & 0,65 & 16,67 & 0,10 & 0 & 0 & 0 & 0 & 0 & 0 & 0 & 0 & 0 & 0 & 0 & 0 \\
\hline Poecilus cupreus L. & 10 & 5,92 & 50,00 & 2,96 & 17 & 10,90 & 50,00 & 5,45 & 29 & 13,88 & 33,34 & 4,62 & 4 & 2,02 & 50,00 & 1,01 & 12 & 5,51 & 50,00 & 2,75 \\
\hline Polydesmus complanatus $L$. & 1 & 0,60 & 16,67 & 0,10 & 2 & 1,29 & 33,34 & 0,43 & 6 & 2,87 & 33,34 & 0,95 & 4 & 2,02 & 33,34 & 0,67 & 5 & 2,30 & 33,34 & 0,76 \\
\hline Pseudoophonus rufipes De Geer & 23 & 13,61 & 83,34 & 11,34 & 11 & 7,06 & 66,67 & 4,70 & 21 & 10,05 & 83,34 & 8,37 & 7 & 3,54 & 50,0 & 1,77 & 14 & 6,43 & 83,34 & 5,35 \\
\hline Pterostichus macer Marsh. & 3 & 1,78 & 16,67 & 0,29 & 4 & 2,57 & 16,67 & 0,42 & 0 & 0 & 0 & 0 & 2 & 1,01 & 16,67 & 0,16 & 1 & 0,46 & 16,67 & 0,07 \\
\hline Pterostichus niger Schall. & 1 & 0,60 & 16,67 & 0,10 & 1 & 0,65 & 16,67 & 0,10 & 0 & 0 & 0 & 0 & 0 & 0 & 0 & 0 & 0 & 0 & 0 & 0 \\
\hline Pterostichus nigrita $F$. & 2 & 1,19 & 33,34 & 0,39 & 6 & 3,85 & 66,67 & 2,56 & 4 & 1,92 & 50,00 & 0,96 & 5 & 2,53 & 33,34 & 0,84 & 0 & 0 & 0 & 0 \\
\hline Pterostichus vulgaris $L$. & 8 & 4,74 & 83,34 & 3,95 & 7 & 4,49 & 66,67 & 2,99 & 8 & 3,83 & 66,67 & 2,55 & 4 & 2,02 & 66,67 & 1,34 & 5 & 2,30 & 50,00 & 1,15 \\
\hline Sarcophaga carnaria L. & 1 & 0,60 & 16,67 & 0,10 & 0 & 0 & 0 & 0 & 1 & 0,48 & 16,67 & 0,08 & 0 & 0 & 0 & 0 & 0 & 0 & 0 & 0 \\
\hline Scelio inermis Zett. & 0 & 0 & 0 & 0 & 0 & 0 & 0 & 0 & 0 & 0 & 0 & 0 & 1 & 0,51 & 16,67 & 0,08 & 0 & 0 & 0 & 0 \\
\hline Scymnus suturalis Thunbg. & 0 & 0 & 0 & 0 & 0 & 0 & 0 & 0 & 0 & 0 & 0 & 0 & 0 & 0 & 0 & 0 & 3 & 1,38 & 16,67 & 0,23 \\
\hline Sphaerius acaroides Waltl. & 0 & 0 & 0 & 0 & 1 & 0,65 & 16,67 & 0,10 & 1 & 0,48 & 16,67 & 0,08 & 1 & 0,51 & 16,67 & 0,08 & 1 & 0,46 & 16,67 & 0,07 \\
\hline Sphaerophoria fatarum Goel. & 1 & 0,60 & 16,67 & 0,10 & 0 & 0 & 0 & $\frac{0}{0}$ & 0 & 0 & 0 & 0 & 0 & 0 & 0 & 0 & 0 & 0 & 0 & 0 \\
\hline Sphaerophoria scripta L. & 1 & 0,60 & 16,67 & 0,10 & 0 & 0 & 0 & 0 & 1 & 0,48 & 16,67 & 0,08 & 0 & 0 & 0 & 0 & 0 & 0 & 0 & 0 \\
\hline Telenomus tetratomus Thoms. & 2 & 1,19 & 16,67 & 0,19 & 3 & 1,93 & 33,34 & 0,64 & 0 & 0 & 0 & 0 & 1 & 0,51 & 16,67 & 0,08 & 2 & 0,92 & 33,34 & 0,30 \\
\hline Trimorus angustipennis Kieff. & 0 & 0 & 0 & 0 & 1 & 0,65 & 16,67 & 0,10 & 0 & 0 & 0 & 0 & 0 & 0 & 0 & 0 & 5 & 2,30 & 66,67 & 1,53 \\
\hline Vespula germanica & 0 & 0 & 0 & 0 & 0 & 0 & 0 & 0 & 2 & 0,96 & 33,34 & 0,32 & 2 & 1,01 & 16,67 & 0,16 & 0 & 0 & 0 & 0 \\
\hline Xantholinus punctulatus Payk. & 1 & 0,60 & 16,67 & 0,10 & 0 & $\frac{0}{0}$ & 0 & 0 & 20 & 0 & $\frac{0}{30,34}$ & $\frac{0,02}{0}$ & $\frac{2}{0}$ & $\frac{1,11}{0}$ & $\frac{10,0}{0}$ & $\frac{0,10}{0}$ & 0 & $\frac{0}{0}$ & 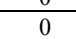 & $\frac{0}{0}$ \\
\hline
\end{tabular}


Table 6. List of the identified taxa and ecological parameters for pest fauna in 2020

\begin{tabular}{|c|c|c|c|c|c|c|c|c|c|c|c|c|c|c|c|c|c|c|c|c|}
\hline \multirow[b]{2}{*}{$\begin{array}{c}\text { Taxa of pest fauna } \\
2020 \\
\end{array}$} & \multicolumn{4}{|l|}{ V1 } & \multicolumn{4}{|l|}{$\mathrm{V} 2$} & \multicolumn{4}{|l|}{ V3 } & \multicolumn{4}{|l|}{$\mathrm{V} 4$} & \multicolumn{4}{|l|}{ V5 } \\
\hline & $\mathrm{A}$ & $\begin{array}{l} \\
(\%)\end{array}$ & $\begin{array}{l}\mathrm{C} \\
(\%)\end{array}$ & $\begin{array}{l}\begin{array}{l}W \\
(\%)\end{array} \\
\end{array}$ & $\mathrm{A}$ & $\begin{array}{l}\text { D } \\
(\%)\end{array}$ & $\begin{array}{l}\mathrm{C} \\
(\%)\end{array}$ & $\begin{array}{l}\mathrm{W} \\
(\%)\end{array}$ & A & $\begin{array}{l} \\
(\%)\end{array}$ & $\begin{array}{l}\mathrm{C} \\
(\%)\end{array}$ & $\begin{array}{l}\mathrm{W} \\
(\%)\end{array}$ & A & $\begin{array}{l}\text { D } \\
(\%)\end{array}$ & $\begin{array}{l}\mathrm{C} \\
(\%)\end{array}$ & $\mathrm{W}(\%)$ & A & $\begin{array}{l}\text { D } \\
(\%)\end{array}$ & $\begin{array}{l}\mathrm{C} \\
(\%)\end{array}$ & $\begin{array}{l}\begin{array}{l}\mathrm{W} \\
(\%)\end{array} \\
(\%)\end{array}$ \\
\hline Adelphocoris seticornis $\mathrm{F}$. & 3 & 0.84 & 45.45 & 0.38 & 0 & 0 & 0 & 0 & 1 & 0.33 & 9.09 & 0.03 & 0 & 0 & 0 & 0 & 1 & 0.35 & 9.09 & 0.03 \\
\hline Agriotes lineatus L. & 0 & 0 & 0 & 0 & 1 & 0.31 & 9.09 & 0.02 & 1 & 0.33 & 9.09 & 0.03 & 0 & 0 & 0 & 0 & 1 & 0.35 & 9.09 & 0.03 \\
\hline Agromyza flaviceps Foel. & 6 & 1.68 & 45.45 & 0.76 & 1 & 0.31 & 9.09 & 0.02 & 1 & 0.33 & 9.09 & 0.03 & 1 & 0.29 & 9.09 & 0.02 & 0 & 0 & 0 & 0 \\
\hline Agromyza nana Meig. & 7 & 1.96 & 36.36 & 0.71 & 24 & 7.54 & 63.63 & 4.79 & 18 & 6.04 & 54.54 & 3.29 & 25 & 7.31 & 72.72 & 5.31 & 24 & 8.57 & 45.45 & 3.89 \\
\hline Aphis fabae Scop. & 0 & 0 & 0 & 0 & 2 & 0.62 & 18.18 & 0.11 & 2 & 0.67 & 18.18 & 0.12 & 0 & 0 & 0 & 0 & 0 & 0 & 0 & 0 \\
\hline Aphrodes bicinctus Schrank & 0 & 0 & 0 & 0 & 0 & 0 & 0 & 0 & 1 & 0.33 & 9.09 & 0.30 & 0 & 0 & 0 & 0 & 0 & 0 & 0 & 0 \\
\hline Austroagallia sinuata Muls. et Rey & 1 & 0.28 & 9.09 & 0.02 & 0 & 0 & 0 & 0 & 0 & 0 & 0 & 0 & 0 & 0 & 0 & 0 & 0 & 0 & 0 & 0 \\
\hline Bruchidius varius Oliv. & 0 & 0 & 0 & 0 & 0 & 0 & 0 & 0 & 0 & 0 & 0 & 0 & 1 & 0.29 & 9.09 & 0.02 & 0 & 0 & 0 & 0 \\
\hline Bryobia rubrioculus Scheut. & 0 & 0 & 0 & 0 & 0 & 0 & 0 & 0 & 0 & 0 & 0 & 0 & 0 & 0 & 0 & 0 & 1 & 0.35 & 9.09 & 0.03 \\
\hline Cacopsylla melanoneura $\mathrm{F}$. & 0 & 0 & 0 & 0 & 0 & 0 & 0 & 0 & 1 & 0.33 & 9.09 & 0.03 & 0 & 0 & 0 & 0 & 0 & 0 & 0 & 0 \\
\hline Chlorops pumilionis Bjerk. & 0 & 0 & 0 & 0 & 0 & 0 & 0 & 0 & 0 & 0 & 0 & 0 & 0 & 0 & 0 & 0 & 1 & 0.35 & 9.09 & 0.03 \\
\hline Crambus perlella Scop. & 0 & 0 & 0 & 0 & 1 & 0.31 & 9.09 & 0.02 & 4 & 1.34 & 36.36 & 0.48 & 0 & 0 & 0 & 0 & 0 & 0 & 0 & 0 \\
\hline Culex pipiens $L$. & 0 & 0 & 0 & 0 & 0 & 0 & 0 & 0 & 0 & 0 & 0 & 0 & 1 & 0.29 & 9.09 & 0.02 & 0 & 0 & 0 & 0 \\
\hline Cydnus aterrimus Forst. & 0 & 0 & 0 & 0 & 2 & 0.62 & 18.18 & 0.11 & 0 & 0 & 0 & 0 & 0 & 0 & 0 & 0 & 0 & 0 & 0 & 0 \\
\hline Delia antiqua Meig. & 0 & 0 & 0 & 0 & 1 & 0.31 & 9.09 & 0.02 & 0 & 0 & 0 & 0 & 0 & 0 & 0 & 0 & 0 & 0 & 0 & 0 \\
\hline Delia platura Meig. & 0 & 0 & 0 & 0 & 0 & 0 & 0 & 0 & 1 & 0.33 & 9.09 & 0.30 & 0 & 0 & 0 & 0 & 0 & 0 & 0 & 0 \\
\hline Diptera-Chloropidae & 0 & 0 & 0 & 0 & 1 & 0.31 & 9.09 & 0.02 & 1 & 0.33 & 9.09 & 0.30 & 4 & 1.17 & 9.09 & 0.10 & 0 & 0 & 0 & 0 \\
\hline Diptera- Culicidae & 3 & 0.84 & 18.18 & 0.15 & 0 & 0 & 0 & 0 & 0 & 0 & 0 & 0 & 0 & 0 & 0 & 0 & 0 & 0 & 0 & 0 \\
\hline Diptera-Muscidae & 0 & 0 & 0 & 0 & 2 & 0.62 & 18.18 & 0.11 & 0 & 0 & 0 & 0 & 2 & 0.58 & 18.18 & 0.10 & 6 & 2.14 & 9.09 & 0.19 \\
\hline Dyspessa ulula Bkh. & 0 & 0 & 0 & 0 & 1 & 0.31 & 9.09 & 0.02 & 0 & 0 & 0 & 0 & 0 & 0 & 0 & 0 & 0 & 0 & 0 & 0 \\
\hline Elachiptera cornuta Fall. & 0 & 0 & 0 & 0 & 5 & 1.57 & 27.27 & 0.42 & 2 & 0.67 & 18.18 & 0.12 & 6 & 1.75 & 18.18 & 0.31 & 0 & 0 & 0 & 0 \\
\hline Gryllus campestris L. & 0 & 0 & 0 & 0 & 0 & 0 & 0 & 0 & 0 & 0 & 0 & 0 & 3 & 0.87 & 18.18 & 0.15 & 1 & 0.35 & 9.09 & 0.03 \\
\hline Haltica oleracea $\mathrm{L}$. & 2 & 0.56 & 18.18 & 0.10 & 0 & 0 & 0 & 0 & 0 & 0 & 0 & 0 & 0 & 0 & 0 & 0 & 0 & 0 & 0 & 0 \\
\hline Helicella itala Linea. & 0 & 0 & 0 & 0 & 0 & 0 & 0 & 0 & 0 & 0 & 0 & 0 & 2 & 0.58 & 18.18 & 0.10 & 1 & 0.35 & 9.09 & 0.03 \\
\hline Heliothrips haemorrhoidalis Bche. & 0 & 0 & 0 & 0 & 0 & 0 & 0 & 0 & 1 & 0.33 & 9.09 & 0.03 & 0 & 0 & 0 & 0 & 0 & 0 & 0 & 0 \\
\hline Liriomyza solani Macq. & 1 & 0.28 & 9.09 & 0.02 & 10 & 3.14 & 27.27 & 0.85 & 3 & 1.00 & 27.27 & 0.27 & 13 & 3.80 & 45.45 & 1.72 & 13 & 4.64 & 36.36 & 1.68 \\
\hline Lygus pratensis $\mathrm{L}$. & 0 & 0 & 0 & 0 & 0 & 0 & 0 & 0 & 0 & 0 & 0 & 0 & 1 & 0.29 & 9.09 & 0.02 & 0 & 0 & 0 & 0 \\
\hline Mamestra oleracea $\mathrm{L}$. & 0 & 0 & 0 & 0 & 0 & 0 & 0 & 0 & 0 & 0 & 0 & 0 & 0 & 0 & 0 & 0 & 1 & 0.35 & 9.09 & 0.03 \\
\hline Melanogryllus desertus Pall. & 0 & 0 & 0 & 0 & 1 & 0.31 & 9.09 & 0.02 & 0 & 0 & 0 & 0 & 0 & 0 & 0 & 0 & 0 & 0 & 0 & 0 \\
\hline Melanotus crassicollis Erich. & 0 & 0 & 0 & 0 & 0 & 0 & 0 & 0 & 0 & 0 & 0 & 0 & 1 & 0.29 & 9.09 & 0.02 & 0 & 0 & 0 & 0 \\
\hline Melighethes aeneus L. & 1 & 0.28 & 9.09 & 0.02 & 3 & 0.94 & 18.18 & 0.17 & 2 & 0.67 & 18.18 & 0.12 & 0 & 0 & 0 & 0 & 0 & 0 & 0 & 0 \\
\hline Meromyza nigriventris Macq. & 2 & 0.56 & 18.18 & 0.10 & 1 & 0.31 & 9.09 & 0.02 & 2 & 0.67 & 18.18 & 0.12 & 7 & 2.04 & 45.45 & 0.92 & 3 & 1.07 & 27.27 & 0.29 \\
\hline Musca domestica $\mathrm{L}$. & 0 & 0 & 0 & 0 & 3 & 0.94 & 18.18 & 0.17 & 0 & 0 & 0 & 0 & 1 & 0.29 & 9.09 & 0.02 & 1 & 0.35 & 9.09 & 0.03 \\
\hline Myzus ornatus Laing. & 1 & 0.28 & 9.09 & 0.02 & 0 & 0 & 0 & 0 & 4 & 1.34 & 18.18 & 0.24 & 3 & 0.87 & 9.09 & 0.07 & 1 & 0.35 & 9.09 & 0.03 \\
\hline Phyllotreta vittula Redtb. & 0 & 0 & 0 & 0 & 1 & 0.31 & 9.09 & 0.02 & 2 & 0.67 & 9.09 & 0.06 & 0 & 0 & 0 & 0 & 0 & 0 & 0 & 0 \\
\hline Phytobia cepae Hend. & 0 & 0 & 0 & 0 & 2 & 0.62 & 9.09 & 0.05 & 1 & 0.33 & 9.09 & 0.03 & 5 & 1.46 & 9.09 & 0.13 & 0 & 0 & 0 & 0 \\
\hline Psammotettix alienus Dahl. & 3 & 0.84 & 36.36 & 0.30 & 4 & 1.25 & 9.09 & 0.11 & 3 & 1.00 & 18.18 & 0.18 & 3 & 0.87 & 9.09 & 0.07 & 5 & 1.78 & 36.36 & 0.64 \\
\hline Psammotettix striatus L. & 12 & 3.37 & 54.54 & 1.83 & 13 & 4.08 & 45.45 & 1.85 & 22 & 7.38 & 54.54 & 4.02 & 15 & 4.38 & 45.45 & 1.99 & 11 & 3.92 & 36.36 & 1.42 \\
\hline Rhinoncus pericarpius $\mathrm{L}$. & 1 & 0.28 & 9.09 & 0.02 & 0 & 0 & 0 & 0 & 0 & 0 & 0 & 0 & 0 & 0 & 0 & 0 & 0 & 0 & 0 & 0 \\
\hline Sitophilus granarius $\mathrm{L}$. & 0 & 0 & 0 & 0 & 0 & 0 & 0 & 0 & 0 & 0 & 0 & 0 & 0 & 0 & 0 & 0 & 1 & 0.35 & 9.09 & 0.03 \\
\hline Tetranychus urticae Koch & 1 & 0.28 & 9.09 & 0.02 & 2 & 0.62 & 9.09 & 0.05 & 0 & 0 & 0 & 0 & 0 & 0 & 0 & 0 & 0 & 0 & 0 & 0 \\
\hline Thrips tabaci Lind. & 0 & 0 & 0 & 0 & 0 & 0 & 0 & 0 & 1 & 0.33 & 9.09 & 0.03 & 0 & 0 & 0 & 0 & 0 & 0 & 0 & 0 \\
\hline
\end{tabular}


Table 7. List of the identified taxa and and ecological parameters for useful fauna in 2020

\begin{tabular}{|c|c|c|c|c|c|c|c|c|c|c|c|c|c|c|c|c|c|c|c|c|}
\hline \multirow{2}{*}{$\begin{array}{l}\text { Taxa of useful fauna } \\
2020\end{array}$} & \multicolumn{4}{|c|}{ V1 } & \multicolumn{4}{|c|}{ V2 } & \multicolumn{4}{|c|}{$\mathrm{V3}$} & \multicolumn{4}{|c|}{$\mathrm{V4}$} & \multicolumn{4}{|c|}{ V5 } \\
\hline & $\mathbf{A}$ & D (\%) & C (\%) & W (\%) & $\mathbf{A}$ & D (\%) & C (\%) & $\mathbf{W}(\%)$ & $\mathbf{A}$ & D (\%) & $\mathrm{C}(\%)$ & $\mathbf{W}(\%)$ & $\mathbf{A}$ & $\mathrm{D}(\%)$ & $\mathrm{C}(\%)$ & $W(\%)$ & $\mathbf{A}$ & $\mathbf{D}(\%)$ & $\mathrm{C}(\%)$ & $\mathbf{W}(\%)$ \\
\hline Acupalpus dorsalis $\mathrm{F}$. & 0 & 0 & 0 & 0 & 0 & 0 & 0 & 0 & 0 & 0 & 0 & 0 & 0 & 0 & 0 & 0 & 1 & 0.35 & 9.09 & 0.03 \\
\hline Acupalpus elegans Panz. & 0 & 0 & 0 & 0 & 0 & 0 & 0 & 0 & 1 & 0.33 & 9.09 & 0.03 & 0 & 0 & 0 & 0 & 1 & 0.35 & 9.09 & 0.03 \\
\hline Acupalpus meridianus L. & 0 & 0 & 0 & 0 & 0 & 0 & 0 & 0 & 0 & 0 & 0 & 0 & 1 & 0.29 & 9.09 & 0.02 & 0 & 0 & 0 & 0 \\
\hline Aleochara pubenula Klug. & 2 & 0.56 & 18.18 & 0.10 & 0 & 0 & 0 & 0 & 0 & 0 & 0 & 0 & 0 & 0 & 0 & 0 & 0 & 0 & 0 & 0 \\
\hline Anchomenus dorsalis Pontopp & 0 & 0 & 0 & 0 & 5 & 1.57 & 9.09 & 0.14 & 0 & 0 & 0 & 0 & 0 & 0 & 0 & 0 & 0 & 0 & 0 & 0 \\
\hline Andrena cineraria Linea. & 1 & 0.28 & 9.09 & 0.02 & 0 & 0 & 0 & 0 & 1 & 0.33 & 9.09 & 0.03 & 0 & 0 & 0 & 0 & 0 & 0 & 0 & 0 \\
\hline Anthicus antherinus L. & 0 & 0 & 0 & 0 & 0 & 0 & 0 & 0 & 1 & 0.33 & 9.09 & 0.03 & 0 & 0 & 0 & 0 & 0 & 0 & 0 & 0 \\
\hline Anthicus floralis $\mathrm{L}$. & 8 & 2.24 & 54.54 & 1.22 & 5 & 1.57 & 36.36 & 0.57 & 9 & 3.02 & 45.45 & 1.37 & 10 & 2.92 & 63.63 & 1.85 & 12 & 4.28 & 36.36 & 1.55 \\
\hline Anthicus quadriguttatus Rossi & 0 & 0 & 0 & 0 & 0 & 0 & 0 & 0 & 0 & 0 & 0 & 0 & 0 & 0 & 0 & 0 & 1 & 0.35 & 9.09 & 0.03 \\
\hline Anthocoris nemorum L. & 0 & 0 & 0 & 0 & 0 & 0 & 0 & 0 & 7 & 2.34 & 45.45 & 1.06 & 1 & 0.29 & 9.09 & 0.02 & 1 & 0.35 & 9.09 & 0.03 \\
\hline Apidae & 0 & 0 & 0 & 0 & 0 & 0 & 0 & 0 & 1 & 0.33 & 9.09 & 0.03 & 0 & 0 & 0 & 0 & 0 & 0 & 0 & 0 \\
\hline Aphelinus flaviventris Kurtj. & 1 & 0.28 & 9.09 & 0.02 & 0 & 0 & 0 & 0 & 1 & 0.33 & 9.09 & 0.03 & 0 & 0 & 0 & 0 & 0 & 0 & 0 & 0 \\
\hline Aphidius colemani Ver. & 0 & 0 & 0 & 0 & 0 & 0 & 0 & 0 & 3 & 1.00 & 18.18 & 0.18 & 1 & 0.29 & 9.09 & 0.02 & 1 & 0.35 & 9.09 & 0.03 \\
\hline Aranea & 109 & 30.61 & 100.0 & 30.61 & 81 & 25.47 & 90.91 & 23.15 & 78 & 26.17 & 100.0 & 26.17 & 69 & 20.17 & 90.90 & 18.33 & 93 & 33.21 & 81.81 & 27.17 \\
\hline Bembidion bipunctatum $\mathrm{L}$. & 0 & 0 & 0 & 0 & 0 & 0 & 0 & 0 & 0 & 0 & 0 & 0 & 1 & 0.29 & 9.09 & 0.02 & 1 & 0.35 & 9.09 & 0.03 \\
\hline Bembidion minimum $\mathrm{F}$. & 0 & 0 & 0 & 0 & 0 & 0 & 0 & 0 & 0 & 0 & 0 & 0 & 0 & 0 & 0 & 0 & 2 & 0.71 & 9.09 & 0.06 \\
\hline Bembidion properans Steph. & 0 & 0 & 0 & 0 & 1 & 0.31 & 9.09 & 0.02 & 1 & 0.33 & 9.09 & 0.03 & 0 & 0 & 0 & 0 & 0 & 0 & 0 & 0 \\
\hline Bolitobius pygmaeus $F$. & 1 & 0.28 & 9.09 & 0.02 & 1 & 0.31 & 9.09 & 0.02 & 0 & 0 & 0 & 0 & 2 & 0.58 & 9.09 & 0.05 & 1 & 0.35 & 9.09 & 0.03 \\
\hline Bradycellus similis Dej. & 0 & 0 & 0 & 0 & 1 & 0.31 & 9.09 & 0.02 & 1 & 0.33 & 9.09 & 0.03 & 2 & 0.58 & 9.09 & 0.05 & 1 & 0.35 & 9.09 & 0.03 \\
\hline Bradysia bicolor Meig. & 0 & 0 & 0 & 0 & 0 & 0 & 0 & 0 & 0 & 0 & 0 & 0 & 1 & 0.29 & 9.09 & 0.02 & 0 & 0 & 0 & 0 \\
\hline Calliphora vicina Rob. Desv. & 0 & 0 & 0 & 0 & 1 & 0.31 & 9.09 & 0.02 & 0 & 0 & 0 & 0 & 0 & 0 & 0 & 0 & 2 & 0.71 & 9.09 & 0.06 \\
\hline Cantharis obscura L. & 0 & 0 & 0 & 0 & 0 & 0 & 0 & 0 & 0 & 0 & 0 & 0 & 0 & 0 & 0 & 0 & 1 & 0.35 & 9.09 & 0.03 \\
\hline Carpophilus sexpustulatus $\mathrm{F}$. & 0 & 0 & 0 & 0 & 0 & 0 & 0 & 0 & 0 & 0 & 0 & 0 & 0 & 0 & 0 & 0 & 2 & 0.71 & 9.09 & 0.06 \\
\hline Ceratophysella bengtssoni Agren & 16 & 4.49 & 90.90 & 4.08 & 26 & 8.17 & 18.18 & 1.48 & 12 & 4.02 & 36.36 & 1.46 & 30 & 8.77 & 45.45 & 3.98 & 3 & 1.07 & 9.09 & 0.09 \\
\hline Cerceris arenaria $\mathrm{L}$. & 0 & 0 & 0 & 0 & 0 & 0 & 0 & 0 & 2 & 0.67 & 9.09 & 0.06 & 0 & 0 & 0 & 0 & 0 & 0 & 0 & 0 \\
\hline Conosoma littoreum $\mathrm{L}$. & 0 & 0 & 0 & 0 & 0 & 0 & 0 & 0 & 0 & 0 & 0 & 0 & 1 & 0.29 & 9.09 & 0.02 & 0 & 0 & 0 & 0 \\
\hline Corticaria crenulata Gyll. & 2 & 0.56 & 18.18 & 0.10 & 0 & 0 & 0 & 0 & 0 & 0 & 0 & 0 & 0 & 0 & 0 & 0 & 0 & 0 & 0 & 0 \\
\hline Corticaria longicollis Zett. & 2 & 0.56 & 18.18 & 0.10 & 0 & 0 & 0 & 0 & 0 & 0 & 0 & 0 & 0 & 0 & 0 & 0 & 0 & 0 & 0 & 0 \\
\hline Corticarina gibbosa Herbst. & 3 & 0.84 & 18.18 & 0.15 & 1 & 0.31 & 9.09 & 0.02 & 1 & 0.33 & 9.09 & 0.03 & 1 & 0.29 & 9.09 & 0.02 & 0 & 0 & 0 & 0 \\
\hline Corticarina truncatella Mnnh. & 2 & 0.56 & 18.18 & 0.10 & 0 & 0 & 0 & 0 & 0 & 0 & 0 & 0 & 1 & 0.29 & 9.09 & 0.02 & 0 & 0 & 0 & 0 \\
\hline Cryptobium fracticorne Payk. & 0 & 0 & 0 & 0 & 0 & 0 & 0 & 0 & 0 & 0 & 0 & 0 & 1 & 0.29 & 9.09 & 0.02 & 0 & 0 & 0 & 0 \\
\hline Dermestes mustelinus Er. & 0 & 0 & 0 & 0 & 0 & 0 & 0 & 0 & 0 & 0 & 0 & 0 & 1 & 0.29 & 9.09 & 0.02 & 0 & 0 & 0 & 0 \\
\hline Diadromus pinplarius Wesm. & 2 & 0.56 & 9.09 & 0.05 & 1 & 0.31 & 9.09 & 0.02 & 0 & 0 & 0 & 0 & 0 & 0 & 0 & 0 & 0 & 0 & 0 & 0 \\
\hline Diptera-Bombyliidae & 0 & 0 & 0 & 0 & 0 & 0 & 0 & 0 & 0 & 0 & 0 & 0 & 1 & 0.29 & 9.09 & 0.02 & 0 & 0 & 0 & 0 \\
\hline Diptera - Conopidae & 0 & 0 & 0 & 0 & 1 & 0.31 & 9.09 & 0.02 & 0 & 0 & 0 & 0 & 1 & 0.29 & 9.09 & 0.02 & 0 & 0 & 0 & 0 \\
\hline Diptera - Sciaridae & 0 & 0 & 0 & 0 & 1 & 0.31 & 9.09 & 0.02 & 2 & 0.67 & 18.18 & 0.12 & 0 & 0 & 0 & 0 & 0 & 0 & 0 & 0 \\
\hline Diptera-Tachinidae & 3 & 0.84 & 27.27 & 0.22 & 3 & 0.94 & 9.09 & 0.08 & 2 & 0.67 & 18.18 & 0.12 & 0 & 0 & 0 & 0 & 0 & 0 & 0 & 0 \\
\hline Drasterius bimaculatus Rossi & 0 & 0 & 0 & 0 & 3 & 0.94 & 27.27 & 0.25 & 4 & 1.34 & 36.36 & 0.48 & 1 & 0.29 & 9.09 & 0.02 & 5 & 1.78 & 36.36 & 0.64 \\
\hline Dromius melanocephalus Dej & 2 & 0.56 & 9.09 & 0.05 & 0 & 0 & 0 & 0 & 0 & 0 & 0 & 0 & 0 & 0 & 0 & 0 & 1 & 0.35 & 9.09 & 0.03 \\
\hline Ephisternus globulus Payk. & 0 & 0 & 0 & 0 & 1 & 0.31 & 9.09 & 0.02 & 0 & 0 & 0 & 0 & 0 & 0 & 0 & 0 & 1 & 0.35 & 9.09 & 0.03 \\
\hline Entomobrya multifasciata Tullb. & 2 & 0.56 & 18.18 & 0.10 & 1 & 0.31 & 9.09 & 0.02 & 0 & 0 & 0 & 0 & 1 & 0.29 & 9.09 & 0.02 & 1 & 0.35 & 9.09 & 0.03 \\
\hline Eugnoriste occidentalis Coq. & 5 & 1.40 & 27.27 & 0.38 & 7 & 2.20 & 45.45 & 1.0 & 8 & 2.68 & 54.54 & 1.46 & 9 & 2.63 & 54.54 & 1.43 & 5 & 1.78 & 36.36 & 0.64 \\
\hline Exorista larvarum $\mathrm{L}$. & 0 & 0 & 0 & 0 & 0 & 0 & 0 & 0 & 0 & 0 & 0 & 0 & 4 & 1.17 & 9.09 & 0.10 & 0 & 0 & 0 & 0 \\
\hline Formica rufa L. & 25 & 7.02 & 63.63 & 4.46 & 18 & 5.66 & 54.54 & 3.08 & 24 & 8.05 & 72.72 & 5.85 & 34 & 9.94 & 63.63 & 6.32 & 23 & 8.21 & 54.54 & 4.47 \\
\hline Gauropterus fulgidus Fr. & 0 & 0 & 0 & 0 & 0 & 0 & 0 & 0 & 1 & 0.33 & 9.09 & 0.03 & 1 & 0.29 & 9.09 & 0.02 & 1 & 0.35 & 9.09 & 0.03 \\
\hline Geocolis grylloides $L$. & 0 & 0 & 0 & 0 & 2 & 0.62 & 18.18 & 0.11 & 0 & 0 & 0 & 0 & 2 & 0.58 & 18.18 & 0.10 & 1 & 0.35 & 9.09 & 0.03 \\
\hline
\end{tabular}




\begin{tabular}{|c|c|c|c|c|c|c|c|c|c|c|c|c|c|c|c|c|c|c|c|c|}
\hline Halictus parallelus Say. & 0 & 0 & 0 & 0 & 1 & 0.31 & \begin{tabular}{|l|}
9.09 \\
\end{tabular} & 0.02 & 0 & 0 & 0 & 0 & 0 & 0 & 0 & 0 & 0 & 0 & 0 & 0 \\
\hline Halyzia 22-punctataL. & 0 & 0 & 0 & 0 & 0 & 0 & 0 & 0 & 3 & 1.00 & 18.18 & \begin{tabular}{|l|}
0.18 \\
\end{tabular} & 0 & 0 & 0 & 0 & 0 & 0 & 0 & 0 \\
\hline Harpalus distinguendus Duft. & 7 & 1.96 & 45.45 & 0.89 & 1 & 0.31 & \begin{tabular}{|l|}
9.09 \\
\end{tabular} & 0.02 & 3 & 1.00 & 18.18 & \begin{tabular}{|l|}
0.18 \\
\end{tabular} & 7 & 2.04 & 27.27 & 0.55 & 0 & 0 & 0 & 0 \\
\hline Harpalus griseus Panz & 3 & 0.84 & 9.09 & 0.07 & 0 & 0 & \begin{tabular}{|c|}
0 \\
\end{tabular} & 0 & 0 & 0 & 0 & 0 & 0 & 0 & 0 & 0 & 0 & 0 & 0 & 0 \\
\hline Hypoaspis aculeifer Canest. & 0 & 0 & 0 & 0 & 3 & 0.94 & 18.18 & 0.17 & 3 & 1.00 & 18.18 & \begin{tabular}{|l|}
0.18 \\
\end{tabular} & 1 & 0.29 & 9.09 & 0.02 & 0 & 0 & 0 & 0 \\
\hline Halictus maculatus Smith. & 0 & 0 & 0 & 0 & $\begin{array}{ll}0 \\
\end{array}$ & 0 & \begin{tabular}{|c|}
0 \\
\end{tabular} & 0 & 2 & $\begin{array}{l}0.67 \\
\end{array}$ & 9.09 & \begin{tabular}{|l|}
0.06 \\
\end{tabular} & 1 & 0.29 & 9.09 & 0.02 & 0 & 0 & 0 & 0 \\
\hline Hormius moniliatusNees & 0 & 0 & 0 & 0 & 0 & 0 & 0 & 0 & 1 & 0.33 & 9.09 & \begin{tabular}{|l|}
0.03 \\
\end{tabular} & 1 & 0.29 & 9.09 & 0.02 & 0 & 0 & 0 & 0 \\
\hline Hybos culiciformis $\mathrm{F}$. & 0 & 0 & 0 & 0 & \begin{tabular}{|c|}
0 \\
\end{tabular} & 0 & 0 & 0 & 0 & 0 & 0 & 0 & 1 & 0.29 & 9.09 & 0.02 & 0 & 0 & 0 & 0 \\
\hline Hymenoptera - Braconidae & 1 & 0.28 & 9.09 & 0.02 & 0 & 0 & 0 & 0 & 1 & 0.33 & 9.09 & \begin{tabular}{|l|}
0.03 \\
\end{tabular} & 0 & 0 & 0 & 0 & 2 & 0.71 & 18.18 & 0.12 \\
\hline Hymenoptera-Ichneumonidae & 0 & 0 & 0 & 0 & 1 & 0.31 & \begin{tabular}{|l|}
9.09 \\
\end{tabular} & 0.02 & 0 & 0 & 0 & \begin{tabular}{|l|l|}
0 \\
\end{tabular} & 0 & 0 & 0 & 0 & 0 & 0 & 0 & 0 \\
\hline Hymenoptera-Scelionidae & 3 & 0.84 & 18.18 & 0.15 & 0 & 0 & 0 & 0 & 2 & 0.67 & 9.09 & \begin{tabular}{|l|}
0.06 \\
\end{tabular} & 0 & 0 & 0 & 0 & 2 & 0.71 & 9.09 & 0.06 \\
\hline Hymenoptera-Sphecidae & 0 & 0 & 0 & 0 & 0 & 0 & 0 & 0 & 0 & 0 & 0 & 0 & 0 & 0 & 0 & 0 & 2 & 0.71 & 9.09 & 0.06 \\
\hline Hypoaspis spp. & 0 & 0 & 0 & 0 & 0 & 0 & 0 & 0 & 0 & 0 & 0 & 0 & 1 & 0.29 & 9.09 & 0.02 & 0 & 0 & 0 & 0 \\
\hline Itoplectis maculatus $\mathrm{F}$. & 2 & 0.56 & 18.18 & 0.10 & 0 & 0 & 0 & 0 & 1 & 0.33 & 9.09 & \begin{tabular}{|l|}
0.03 \\
\end{tabular} & 0 & 0 & 0 & 0 & 0 & 0 & 0 & 0 \\
\hline Kleidotoma brunnea Ion. & 3 & 0.84 & 18.18 & 0.15 & 2 & 0.62 & \begin{tabular}{|l|}
18.18 \\
\end{tabular} & 0.11 & 4 & 1.34 & 18.18 & \begin{tabular}{|l|}
0.24 \\
\end{tabular} & 4 & 1.17 & 18.18 & 0.21 & 1 & 0.35 & 9.09 & 0.03 \\
\hline Lasius flavus $\mathrm{F}$. & 11 & 3.09 & 27.27 & 0.84 & 8 & 2.51 & \begin{tabular}{|l|l}
54.54 \\
\end{tabular} & 1.36 & 11 & 3.69 & 45.45 & \begin{tabular}{|l|}
1.67 \\
\end{tabular} & 10 & 2.92 & 63.63 & 1.85 & 12 & 4.28 & 54.54 & 2.33 \\
\hline Lasius niger $\mathrm{L}$. & 0 & 0 & 0 & 0 & \begin{tabular}{|c|}
0 \\
\end{tabular} & 0 & 0 & 0 & 1 & 0.33 & 9.09 & \begin{tabular}{|l|}
0.03 \\
\end{tabular} & 0 & 0 & 0 & 0 & 0 & 0 & 0 & 0 \\
\hline Lithobius forficatus $\mathrm{L}$. & 0 & 0 & 0 & 0 & 0 & 0 & 0 & 0 & 0 & 0 & 0 & 0 & 3 & 0.87 & 18.18 & 0.15 & 1 & 0.35 & 9.09 & 0.03 \\
\hline Longitarsus gracilis Kutsch. & 0 & 0 & 0 & 0 & 0 & 0 & 0 & 0 & 0 & 0 & 0 & 0 & 0 & 0 & 0 & 0 & 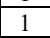 & 0.35 & 9.09 & 0.03 \\
\hline Longitarsus nasturtii $\mathrm{F}$. & 0 & 0 & 0 & 0 & \begin{tabular}{|c|}
0 \\
\end{tabular} & 0 & 0 & 0 & 0 & 0 & 0 & 0 & 0 & 0 & 0 & 0 & 1 & 0.35 & 9.09 & 0.03 \\
\hline Medetera tristis Zett. & 0 & 0 & 0 & 0 & 0 & 0 & 0 & 0 & 0 & 0 & 0 & 0 & 1 & 0.29 & 9.09 & 0.02 & 0 & 0 & 0 & 0 \\
\hline Microlestes maurus Strm. & 0 & 0 & 0 & 0 & \begin{tabular}{|c|}
0 \\
\end{tabular} & 0 & 0 & 0 & 1 & 0.33 & 9.09 & \begin{tabular}{|l|}
0.03 \\
\end{tabular} & 0 & 0 & 0 & 0 & 0 & 0 & 0 & 0 \\
\hline Microlestes plagiatus Duft. & 0 & 0 & 0 & 0 & 0 & 0 & 0 & 0 & 1 & 0.33 & 9.09 & \begin{tabular}{|l|}
0.03 \\
\end{tabular} & 1 & 0.29 & 9.09 & 0.02 & \begin{tabular}{|lll}
1 \\
\end{tabular} & 0.35 & 9.09 & 0.03 \\
\hline Myrmica rubra L. & 12 & 3.37 & 54.54 & 1.83 & 2 & 0.62 & 18.18 & 0.11 & 5 & 1.67 & 18.18 & 0.30 & 2 & 0.58 & 18.18 & 0.10 & 0 & 0 & 0 & 0 \\
\hline Nabis ferus L. & 4 & 1.12 & 27.27 & 0.30 & 1 & 0.31 & \begin{tabular}{|l|}
9.09 \\
\end{tabular} & 0.02 & 0 & 0 & 0 & 0 & 1 & 0.29 & 9.09 & 0.02 & 0 & 0 & 0 & 0 \\
\hline Ocyusa incrasata Rey. & 0 & 0 & 0 & 0 & 0 & 0 & \begin{tabular}{|c|c|}
0 \\
\end{tabular} & 0 & 0 & 0 & 0 & 0 & 1 & 0.29 & 9.09 & 0.02 & 0 & 0 & 0 & 0 \\
\hline Ophonus sabulicola Panz. & 0 & 0 & 0 & 0 & 0 & 0 & 0 & 0 & 0 & 0 & 0 & 0 & 0 & 0 & 0 & 0 & 1 & 0.35 & 9.09 & 0.03 \\
\hline Orius niger Wolff. & 2 & 0.56 & 18.18 & 0.10 & 0 & 0 & 0 & 0 & 0 & 0 & 0 & 0 & 0 & 0 & 0 & 0 & 1 & 0.35 & 9.09 & 0.03 \\
\hline Oxypoda togata Er. & 0 & 0 & 0 & 0 & 0 & 0 & 0 & 0 & 0 & 0 & 0 & 0 & 1 & 0.29 & 9.09 & 0.02 & 0 & 0 & 0 & 0 \\
\hline Phalangium opilio $\mathrm{L}$. & 12 & 3.37 & 45.45 & 1.53 & 0 & 0 & 0 & 0 & 1 & 0.33 & 9.09 & \begin{tabular}{|l|}
0.03 \\
\end{tabular} & 0 & 0 & 0 & 0 & 0 & 0 & 0 & 0 \\
\hline Phygadeuon fumator Grav. & 0 & 0 & 0 & 0 & 0 & 0 & 0 & 0 & 0 & 0 & 0 & \begin{tabular}{|l|l|}
0 \\
\end{tabular} & 1 & 0.29 & 9.09 & 0.02 & 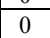 & 0 & 0 & 0 \\
\hline Picromerus bidens $L$. & 0 & 0 & 0 & 0 & 0 & 0 & 0 & 0 & 0 & 0 & 0 & 0 & 1 & 0.29 & 9.09 & 0.02 & 0 & 0 & 0 & 0 \\
\hline Pleurophorus caesus Panz. & 0 & 0 & 0 & 0 & 0 & 0 & \begin{tabular}{|c|c|c|}
0 \\
\end{tabular} & 0 & 0 & 0 & 0 & \begin{tabular}{|c|c|}
0 \\
\end{tabular} & 1 & 0.29 & 9.09 & 0.02 & 22 & 0.71 & 18.18 & 0.12 \\
\hline Poecilus cupreus L. & 6 & 1.68 & 36.36 & 0.61 & 2 & 0.62 & \begin{tabular}{|l|l|}
18.18 \\
\end{tabular} & 0.11 & 1 & 0.33 & 9.09 & \begin{tabular}{|l|}
0.03 \\
\end{tabular} & 0 & 0 & 0 & 0 & 0 & 0 & 0 & 0 \\
\hline Polydesmus complanatus $\mathrm{L}$. & 0 & 0 & 0 & 0 & 2 & 0.62 & 9.09 & 0.05 & 1 & 0.33 & 9.09 & \begin{tabular}{|l|}
0.03 \\
\end{tabular} & 1 & 0.29 & 9.09 & 0.02 & 1 & 0.35 & 9.09 & 0.03 \\
\hline Porcellio scaber $\mathrm{L}$. & 0 & 0 & 0 & 0 & 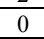 & 0 & \begin{tabular}{|c|c|}
0 \\
\end{tabular} & 0 & \begin{tabular}{|c|}
0 \\
\end{tabular} & 0 & 0 & \begin{tabular}{|c|}
0 \\
\end{tabular} & 0 & 0 & 0 & 0 & 1 & 0.35 & 9.09 & 0.03 \\
\hline Pterostichus niger Schall. & 0 & 0 & 0 & 0 & 5 & 1.57 & \begin{tabular}{|l|}
27.27 \\
\end{tabular} & 0.42 & 3 & 1.00 & 27.27 & \begin{tabular}{|l|}
0.27 \\
\end{tabular} & 4 & 1.17 & 27.27 & 0.31 & 6 & 2.14 & 18.18 & 0.38 \\
\hline Pterostichus vulgaris L. & 8 & 2.24 & 45.45 & 1.01 & 1 & 0.31 & \begin{tabular}{|l|l|}
9.09 \\
\end{tabular} & 0.02 & \begin{tabular}{|c|}
1 \\
\end{tabular} & 1.00 & 9.09 & \begin{tabular}{|l|}
0.09 \\
\end{tabular} & 0 & 0 & 0 & 0 & \begin{tabular}{|l|l|l|}
0 \\
\end{tabular} & 0 & 0 & 0 \\
\hline Pseudoophonus rufipes De Geer & 49 & 13.77 & 100 & 13.77 & 26 & 8.17 & 72.72 & 5.94 & \begin{tabular}{|c|}
7 \\
\end{tabular} & 2.34 & 36.36 & \begin{tabular}{|c|}
0.85 \\
\end{tabular} & 13 & 3.80 & 72.72 & 2.76 & 10 & 3.57 & 27.27 & 0.97 \\
\hline Sarcophaga carnaria $\mathrm{L}$. & 0 & 0 & 0 & 0 & 16 & 5.03 & 36.36 & 1.82 & \begin{tabular}{|c|}
0 \\
\end{tabular} & 0 & 0 & 0 & 6 & 1.75 & 36.36 & 0.63 & \begin{tabular}{|c|c|}
0 \\
\end{tabular} & 0 & 0 & 0 \\
\hline Sceliphron spirifex Line. & 1 & 0.28 & 9.09 & 0.02 & 0 & 0 & \begin{tabular}{|l|l|l}
0 & \\
\end{tabular} & 0 & \begin{tabular}{|c|c|}
1 & \\
\end{tabular} & 0.33 & 9.09 & \begin{tabular}{|l|}
0.03 \\
\end{tabular} & 0 & 0 & 0 & 0 & 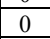 & 0 & 0 & 0 \\
\hline Sciara analis Schin. & 2 & 0.56 & 18.18 & 0.10 & 2 & 0.62 & \begin{tabular}{|l|l|}
9.09 \\
\end{tabular} & 0.05 & 2 & 0.67 & 9.09 & 0.06 & 1 & 0.29 & 9.09 & 0.02 & 0 & 0 & 0 & 0 \\
\hline Scopeus cognatus Rey. & 0 & 0 & 0 & 0 & 0 & 0 & 0 & 0 & 0 & 0 & 0 & 0 & 0 & 0 & 0 & 0 & 1 & 0.35 & 9.09 & 0.03 \\
\hline Stenichneumon culpator Schrank & 0 & 0 & 0 & 0 & 1 & 0.31 & 9.09 & 0.02 & 2 & 0.67 & 9.09 & 0.06 & 2 & 0.58 & 9.09 & 0.05 & 1 & 0.35 & 9.09 & 0.03 \\
\hline Tachina fallax Meig. & 0 & 0 & 0 & 0 & 3 & 0.94 & 18.18 & 0.17 & 6 & 2.01 & 27.27 & 0.54 & 4 & 1.17 & 27.27 & 0.31 & 0 & 0 & 0 & 0 \\
\hline Trissolcus semistriatus Ness. & 0 & 0 & 0 & 0 & 0 & 0 & 0 & 0 & 0 & 0 & 0 & 0 & 1 & 0.29 & 9.09 & 0.02 & 0 & 0 & 0 & 0 \\
\hline
\end{tabular}


The species and group of species that reached the maximum values of the total fauna both useful and harmful in the 2 years of survey are presented in Table 8 .

Table 8. Values of ecological parameters for the most abundant fauna

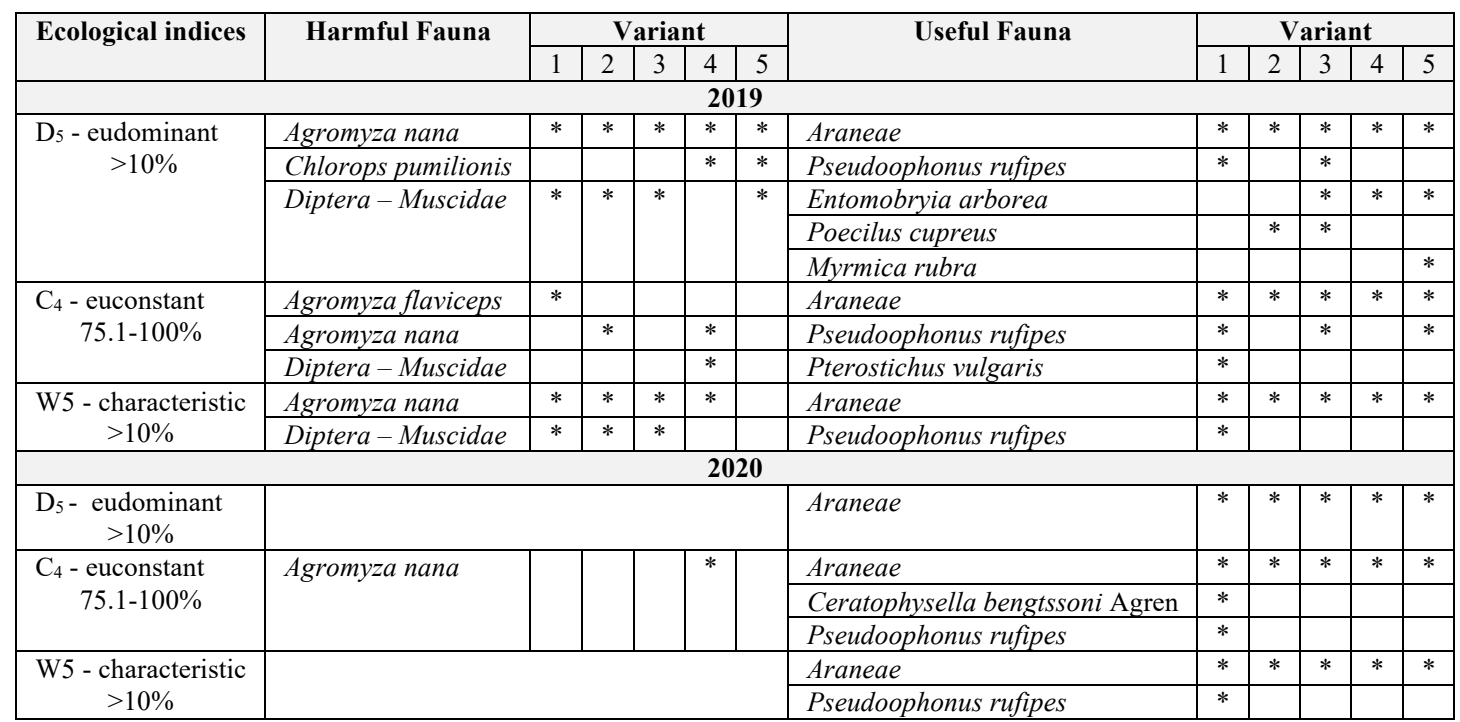

Among useful fauna, different species from the Araneae order have been found. The great majority of them feed on insects and they can be found with a high species diversity in almost all habitats and microhabitats, from subterranean environments to mountain tops but also in agricultural areas (Marc et al., 1999).

Another important group of useful arthropods with a high abundance was Collembola, represented by two species Entomobryia arborea and Ceratophysella bengtssoni. Collembola species indirectly contribute in the process of decomposition through the fragmentation of organic matter and the control of soil microbial communities (Brady \& Weil, 2009). They are common in both natural and anthropogenic habitats (Babenko et al., 1994).

Concerning insects in Table 8, the Coleoptera order consisted of three useful species from the Carabidae family: Pseudoophonus rufipes, Poecilus cupreus and Pterostichus vulgaris. They are very common polyphagous predatory species found in agroecosystems and are considered to play an important role in the natural control of pests (Cavaliere, 2019). Another order containing useful species was Hymenoptera, with the most numerous specimens in our study belonging to the ant Myrmica rubra. This species is a generalist omnivore with a wide food spectrum that includes other insects, pollen as well as honeydew and nectar (Reznikova \& Panteleeva, 2001; Ness et al., 2013). The preferred habitat of $M$. rubra are gardens and agroecosystems (Michlewicz \& Tryjanowski, 2017).

The harmful fauna with the most abundant specimens was represented by Diptera order, the main representatives being the species Agromyza nana, A. flaviceps and Chlorops pumilionis to which was added the Muscidae family. Larvae of Agromyzidae and Chloropidae are pest of agricultural plants and weeds (Kaniuczak, 2011; Pap, 1984). Members of Muscidae family are hematophagous parasites, vectors of different diseases and pests to humans and domesticated animals and they exploit foods and various habitats such as agroecosystems (Moon, 2019).

\section{ACKNOWLEDGEMENTS}

This work was supported by a grant of the Romanian Ministry of Research and Innovations, CCCDIUEFISCDI, project number PN-III-P1-1.2-PCCDI-2017-0659 PCCDI, within PNCDI III. 


\section{CONCLUSION}

Data from the present study indicated that applications of diatomite from Pătârlagele deposit (Buzău County) quantitatively reduced epigeal fauna in pepper crops while the applications of bioinoculant based on Trichoderma asperellum Td85 favored it. Further new field studies are necessary to be developed in the near future to evaluate the effects of the two products.

\section{REFERENCES}

BABAN, E. (2006). Diversitatea coleopterelor (Coleoptera: Carabidae, Silphidae, Scarabaeidae, Cerambycidae) din ecosistemele forestiere ale Podişului Moldovei Centrale. Academia de Științe a Moldovei, Institutul de Zoologie, 136.

BABENKO, A. B., CHERNOVA, N. M., POTAPOV, M. B., STEBAEVA, S. K. (1994). Collembola of Russia and adjacent countries: Family Hypogastruridae. Nauka, Moscow, 336. BARDGETT, R. D., COOK, R. (1998). Functional aspects of soil animal diversity in agricultural grasslands. Applied Soil Ecology, 10, 263-276.

BRADY, N. C., WEIL, R. R. (2009). Organisms and ecology of the soil. Elements of the nature and properties of soils (3rd ed.). Upper Saddle River: Prentice Hall, 965.

BUCUR, A., ROȘCA, I. (2011). Research regarding biology of rape pests. Scientific Papers, UASVM Bucharest, Series A, 54, 356-359.

BUŞMACHIU, G., BACAL, S. (2012). The contributions to the knowledge of invertebrates (Collembola; Insecta: Coleoptera) from three different crops. Oltenia. Studii şi Comunicări, Ştiinţele Naturii, 28, 1, 49-54.

CAVALIERE, F., BRANDMAYR, P., GIGLIO, A. (2019). DNA damage in haemocytes of Harpalus (Pseudophonus) rufipes (De Geer, 1774) (Coleoptera, Carabidae) as an indicator of sublethal effects of exposure to herbicides. Ecological Indicators, 98, 88-91.

COYLE, D. R., NAGENDRA, U. J., TAYLOR, M. K., CAMPBELL, J. H., CUNARD, C. E., JOSLIN, A. H., MUNDEPI, A., PHILlIPS, C. A., CALLAHAM, JR. M. A. (2017). Soil fauna responses to natural disturbances, invasive species, and global climate change: Current state of the science and a call to action. Soil Biology \& Biochemistry, 110, 116-133.

GILlER, K. E., BEARE, M. H., LAVELLE, P., IZAC, A.-M. N., SWIFT, M. J. (1997). Agricultural intensification, soil biodiversity and agroecosystem function. Applied Soil Ecology, 6, 3-16.

KARUPPAIAH, V., SUJIYANAD, G. K. (2012). Impact of climate change on population dynamics of insect's pests. World Journal of Agricultural Sciences, 8, 3, 240-246.

KANIUCZAK, Z. (2008). Distribution and effects of chemical control of gout fly (Chlorops pumilionis Bjerk.) on spring wheat in south-eastern Poland. Journal of plant protection research. 48, 4, 453-460.

MANOLE, L., TĂLMACIU, M., TĂLMACIU, N. (2009). Some aspects on the structure and abundance of species coleoptere for rapeseed crop-autumn. Analele Universitâții din Craiova, Seria Agriculturã-Montanologie-Cadastru, 39, 216-222.

MARC, P., CANARD, A., YSNE, F. (1999). Spiders (Araneae) useful for pest limitation and bioindication. Invertebrate Biodiversity as Bioindicators of Sustainable Landscapes. Agriculture, Ecosystems and Environment, 74, 229-273.

MICHLEWICZ, M., TRYJANOWSKI, P. (2017). Anthropogenic waste products as preferred nest sites for Myrmica rubra (L.) (Hymenoptera, Formicidae). Journal of Hymenoptera Research, 57, 103-114. 
MOCANU, I., TĂLMACIU, M., TĂLMACIU, N. (2017). The structure and abundance of invertebrate fauna in wheat crop. Current Trends in Natural Sciences, 6, 12, 190-196.

NESS, J. H., MORALES, M. A., KENISON, E., LEDUC, E., LEIPZIG-SCOTT, P., ROLLINSON, E., SWIMM, B. J., VON ALLMEN, D. R. (2013). Reciprocally beneficial interactions between introduced plants and ants are induced by the presence of a third introduced species. Oikos, 122, 695-704.

MOON, R. D. (2019). Muscid Flies (Muscidae). Medical and Veterinary Entomology, 345368.

PAP, L. (1984). Notes on the bionomics of Agromyza nana Meigen (Dipt., Agromyzidae) in Hungary. Journal of applied entomology, 98, 1-5, 280-286.

REZNIKOVA, Z. I., PANTELEEVA, S. N. (2001). Interaction of the ant Myrmica rubra L. as a predator with springtails (Collembola) as a mass prey. Doklady Biological Sciences, 380, 475-477.

ROTARI, E. (2012). Cercetări cu privire la fauna dăunătoare şi utilă din unele culturi de plante legumicole în funcţie de tratamentele chimice aplicate în combaterea dăunătorilor. $\mathrm{PhD}$ thesis.

SANGLE, P. M., SATPUTE, S. B., KHAN, F. S., RODE, N. S. (2015). Impact of climate change on Insects. Trends in Biosciences, 8, 14, 3579-3582.

STANCĂ-MOISE, C. (2019). The insects abundance monitoring in a meadow from Marita village (Vâlcea County, Romania). Studia Universitatis "Vasile Goldiş “, 29, 3, 106-113.

VARVARA, M., GĂLUȘCĂ, S. (2007). Diversity and ecological aspects of the species of Carabidae (Coleoptera, Carabidae) in the sugar beet crop ecosystem from the locality of Trușești (Botoșani County). Muzeul Olteniei Craiova. Oltenia. Studii și Comunicări. Stiințele Naturii, 23, 125-133.

VARVARA, M. (2016). Distribution, Abundance and Dominance of Three Brachinus Species (Coleoptera: Carabidae) in Seven Agricultural Crops in Romania, within the Period 19772010. Travaux du Muséum National d'Histoire Naturelle Grigore Antipa, 59, 2, 161-178.

VOICU, M., MATEIAŞ, T., SĂPUNARU, T. (1983). Insecte parazite şi prădătoare ale dăunătorilor din culturile de lucernă. Probleme de Protecţia Plantelor, 11, 2, 135-145. 